\title{
Transformations of the transfinite plane
}

\author{
Assaf Rinot and Jing Zhang \\ Department of Mathematics, Bar-Ilan University, Ramat-Gan 5290002, Israel; E-mail: rinotas@ math.biu.ac.il, \\ jingzhangjz13@gmail.com.
}

Received: 30 March 2020; Accepted: 22 January 2021

2020 Mathematics Subject Classification: Primary - 03E02; Secondary - 03 E35

\begin{abstract}
We study the existence of transformations of the transfinite plane that allow one to reduce Ramsey-theoretic statements concerning uncountable Abelian groups into classical partition relations for uncountable cardinals.

To exemplify: we prove that for every inaccessible cardinal $\kappa$, if $\kappa$ admits a stationary set that does not reflect at inaccessibles, then the classical negative partition relation $\kappa \rightarrow[K]_{K}^{2}$ implies that for every Abelian group $(G,+)$ of size $\kappa$, there exists a map $f: G \rightarrow G$ such that for every $X \subseteq G$ of size $\kappa$ and every $g \in G$, there exist $x \neq y$ in $X$ such that $f(x+y)=g$.
\end{abstract}

\section{Introduction}

Ramsey's theorem [Ram30] asserts that every infinite graph contains an infinite induced subgraph that is either a clique or an anti-clique. In other words, for every function (or coloring, or partition, depending on one's perspective) $c:[\mathbb{N}]^{2} \rightarrow 2$, there exists an infinite $X \subseteq \mathbb{N}$ that is monochromatic in the sense that, for some $i \in 2, c(x, y)=i$ for every pair $x<y$ of elements of $X$. A strengthening of Ramsey's theorem due to Hindman [Hin74] concerns the additive structure $(\mathbb{N},+)$ and asserts that for every partition $c: \mathbb{N} \rightarrow 2$, there exists an infinite $X \subseteq \mathbb{N}$ that is monochromatic in the sense that, for some $i \in 2$, for every finite increasing sequence $x_{0}<\cdots<x_{n}$ of elements of $X, c\left(x_{0}+\cdots+x_{n}\right)=i$.

A natural generalization of Ramsey's and Hindman's theorems would assert that in any 2-partition of an uncountable structure, there must exist an uncountable monochromatic subset. However, this is not the case. In the early 1930s, Sierpiński found a coloring $c:[\mathbb{R}]^{2} \rightarrow 2$ admitting no uncountable monochromatic set [Sie33]. In contrast, a counterexample concerning the additive structure $(\mathbb{R},+)$ was discovered only a few years ago [HLS17], by Hindman, Leader and Strauss.

In this paper, we study the existence of transformations of the transfinite plane that allow one, among other things, to reduce the additive problem into the considerably simpler Ramsey-type problem.

Throughout the paper, $\kappa$ denotes a regular uncountable cardinal, and $\theta, \chi$ denote (possibly finite) cardinals $\leq \kappa$. The following definition captures the class of transformations of interest.

Definition 1.1. See Figure 1 below. $\mathrm{P} \ell_{1}(\kappa)$ asserts the existence of a transformation $\mathbf{t}:[\kappa]^{2} \rightarrow[\kappa]^{2}$ satisfying the following:

○ For every $(\alpha, \beta) \in[\kappa]^{2}$, if $\mathbf{t}(\alpha, \beta)=\left(\alpha^{*}, \beta^{*}\right)$, then $\alpha^{*} \leq \alpha<\beta^{*} \leq \beta$.

- For every family $\mathcal{A}$ consisting of $\kappa$ many pairwise disjoint finite subsets of $\kappa$, there exists a stationary $S \subseteq \kappa$ such that for every pair $\alpha^{*}<\beta^{*}$ of elements of $S$, there exists a pair $a<b$ of elements of $\mathcal{A}$ with $\mathbf{t}[a \times b]=\left\{\left(\alpha^{*}, \beta^{*}\right)\right\}$.

(C) The Author(s), 2021. Published by Cambridge University Press. This is an Open Access article, distributed under the terms of the Creative Commons Attribution licence (http://creativecommons.org/licenses/by/4.0/), which permits unrestricted re-use, distribution, and reproduction in any medium, provided the original work is properly cited. 

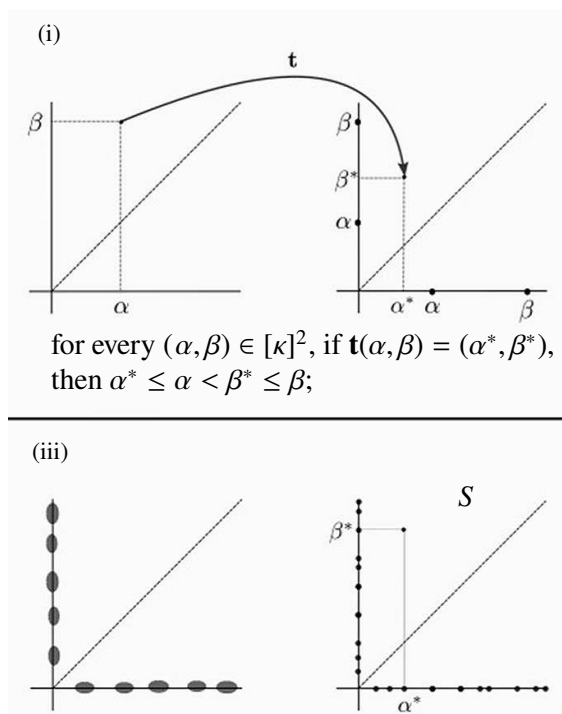

there exists a stationary $S \subseteq \kappa$ such that, for every pair $\alpha^{*}<\beta^{*}$ of elements of $S$, (ii)
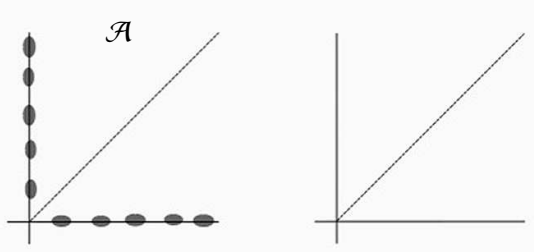

for every family $\mathcal{A}$ consisting of $\kappa$ many pairwise disjoint finite subsets of $\kappa$,

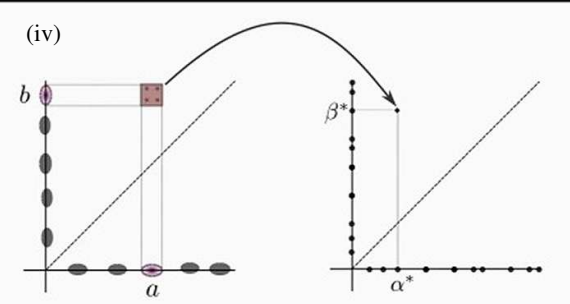

there exists a pair $a<b$ of elements of $\mathcal{A}$ with $\mathbf{t}[a \times b]=\left\{\left(\alpha^{*}, \beta^{*}\right)\right\}$.

Figure 1. Illustration of Definition 1.1.

Theorem A. If $\mathrm{P} \ell_{1}(\kappa)$ holds, then the following are equivalent:

- There exists a coloring $c:[\kappa]^{2} \rightarrow \theta$ such that for every $X \subseteq \kappa$ of size $\kappa$ and every $\tau \in \theta$, there exist $x \neq y$ in $X$ such that $c(x, y)=\tau$;

- For every Abelian group $(G,+)$ of size $\kappa$, there exists a coloring $c: G \rightarrow \theta$ such that for all $X, Y \subseteq G$ of size $\kappa$ and every $\tau \in \theta$, there exist $x \in X$ and $y \in Y$ such that $c(x+y)=\tau$.

As the proof of Theorem A will make clear, the theorem remains valid even after relaxing Definition 1.1 to omit the first bullet and to weaken "stationary $S \subseteq \kappa$ " into "cofinal $S \subseteq \kappa$ ". We have added these extra requirements to connect this line of investigation with other well-known problems, such as the problem of whether the product of any two $\kappa$-cc posets must be $\kappa$-cc (see [Rin14a]):

Theorem B. If $\mathrm{P} \ell_{1}(\kappa)$ holds, then for every positive integer $n$ there exists a poset $\mathbb{P}$ such that $\mathbb{P}^{n}$ satisfies the $\kappa$-cc, but $\mathbb{P}^{n+1}$ does not.

Now, to formulate the main results of this paper, let us consider a more informative variation of $\mathrm{P} \ell_{1}(\kappa)$.

Definition 1.2. $\mathrm{P} \ell_{1}(\kappa, \theta, \chi)$ asserts the existence of a function $\mathbf{t}:[\kappa]^{2} \rightarrow[\kappa]^{3}$ satisfying the following:

$\circ$ For all $(\alpha, \beta) \in[\kappa]^{2}$, if $\mathbf{t}(\alpha, \beta)=\left(\tau^{*}, \alpha^{*}, \beta^{*}\right)$, then $\tau^{*} \leq \alpha^{*} \leq \alpha<\beta^{*} \leq \beta$.

○ For all $\sigma<\chi$ and every family $\mathcal{A} \subseteq[\kappa]^{\sigma}$ consisting of $\kappa$ many pairwise disjoint sets, there exists a stationary $S \subseteq \kappa$ such that for all $\left(\alpha^{*}, \beta^{*}\right) \in[S]^{2}$ and $\tau^{*}<\min \left\{\theta, \alpha^{*}\right\}$, there exist $(a, b) \in[\mathcal{A}]^{2}$ with $\mathbf{t}[a \times b]=\left\{\left(\tau^{*}, \alpha^{*}, \beta^{*}\right)\right\}$.

Remark 1.3. $\mathrm{P} \ell_{1}(\kappa)$ of Definition 1.1 is $\mathrm{P} \ell_{1}\left(\kappa, 1, \boldsymbol{\aleph}_{0}\right)$.

In [Rin12], by building on the work of Eisworth in [Eis13a, Eis13b], the first author proved that $\mathrm{P} \ell_{1}\left(\lambda^{+}, \operatorname{cf}(\lambda), \operatorname{cf}(\lambda)\right)$ holds for every singular cardinal $\lambda .{ }^{1}$ The proof of that theorem was a combination of walks on ordinals, club-guessing considerations, applications of elementary submodels, and oscillation

${ }^{1}$ The first bullet of Definition 1.2 is not stated explicitly but may be verified to hold in all the relevant arguments of [Eis13a, Eis13b, Rin12]. 
of $p c f$ scales. Here, we replace the last ingredient with the oscillation oracle $\mathrm{P} \ell_{6}(\ldots)$ from [Rin $\left.14 \mathrm{~b}\right]$; there are a few additional differences that are too technical to state at this point.

The main result of this paper is as follows:

Theorem C. For $\chi=\mathrm{cf}(\chi) \geq \omega, \mathrm{P} \ell_{1}(\kappa, \theta, \chi)$ holds in any of the following cases:

1. $\chi<\chi^{+}<\theta=\kappa$, and $\square(\kappa)$ holds.

2. $\chi<\chi^{+}<\theta=\kappa$, and $E_{\geq \chi}^{\kappa}$ admits a stationary set that does not reflect.

3. $\chi<\chi^{+}=\theta<\kappa, \kappa$ is inaccessible, and $E_{\geq \chi}^{\kappa}$ admits a stationary set that does not reflect at inaccessibles.

By the results of Subsection 2.3 below, the principle $\mathrm{P} \ell_{1}(\kappa, \theta, \chi)$ is strictly stronger than Shelah's principle $\operatorname{Pr}_{1}(\kappa, \kappa, \theta, \chi)$. Thus, Clause (1) improves the main result of [Rin14a], and Clause (2) improves the main result of [Rin14b]. The result of Clause (3) provides, in particular, an affirmative answer to a question posed by Eisworth to the first author at the Set Theory meeting in Oberwolfach, January 2014.

We conclude the introduction by mentioning two findings in the other direction.

Theorem D. For a strongly inaccessible cardinal $\kappa$ :

1. The existence of a coherent $\kappa$-Souslin tree does not imply $\mathrm{P} \ell_{1}(\kappa)$.

2. For any $\chi \in \operatorname{Reg}(\kappa)$, the existence of a nonreflecting stationary subset of $E_{\chi}^{\kappa}$ does not imply $\mathrm{P} \ell_{1}\left(\kappa, 1, \chi^{+}\right)$.

\subsection{Organization of this paper}

In Section 2, we establish some facts about walks on ordinals and present a connection between $\mathrm{P} \ell_{1}(\kappa, \ldots)$ and two other concepts: the coloring principle $\operatorname{Pr}_{1}(\kappa, \ldots)$ and the $C$-sequence number, $\chi(\kappa)$. The proofs of Theorems A, B and $D$ will be found there.

In Section 3, we prove that a strong form of the oscillation oracle $\mathrm{P} \ell_{6}\left(v^{+}, v\right)$ holds for any infinite regular cardinal $v$. This fact will play a role in later sections.

In Section 4, we provide a proof of Clause (2) of Theorem C. The proof is split into two cases: $\kappa>\chi^{++}$and $\kappa=\chi^{++}$.

In Section 5, we provide a proof of Clause (1) of Theorem C.

In Section 6, we provide a proof of Clause (3) of Theorem C.

\subsection{Further results}

In an upcoming paper [RZ21], we address the validity of the strongest possible instances of $\mathrm{P} \ell_{1}(\kappa, \theta, \chi)$. Some of the main findings are:

- $\mathrm{P} \ell_{1}\left(\lambda^{+}, 1, \lambda\right)$ fails for $\lambda$ singular, so that Theorem $\mathrm{C}$ is optimal whenever $\kappa$ is a successor of a singular cardinal.

$\circ \mathrm{P} \ell_{1}\left(\lambda^{+}, 1, \lambda^{+}\right)$fails for $\lambda$ regular.

$\circ \mathrm{P} \ell_{1}\left(\lambda^{+}, \lambda^{+}, \lambda\right)$ holds for $\lambda$ regular satisfying $2^{\lambda}=\lambda^{+}$.

- $\mathrm{P} \ell_{1}\left(\boldsymbol{\aleph}_{1}, \boldsymbol{\aleph}_{1}, n\right)$ holds for all positive integers $n$.

$\circ \mathrm{P} \ell_{1}(\kappa, \kappa, \kappa)$ holds for $\kappa$ inaccessible such that $\square(\kappa)$ and $\diamond^{*}(\kappa)$ both hold.

\subsection{Notation and conventions}

Let $E_{\chi}^{\kappa}:=\{\alpha<\kappa \mid \operatorname{cf}(\alpha)=\chi\}$, and define $E_{\leq \chi}^{\kappa}, E_{<\chi}^{\kappa}, E_{\geq \chi}^{\kappa}, E_{>\chi}^{\kappa}, E_{\neq \chi}^{\kappa}$ analogously. For an ideal $\mathcal{I}$ over $\kappa$, we write $\mathcal{I}^{+}:=\mathcal{P}(\kappa) \backslash \mathcal{I}$. The collection of all sets of hereditary cardinality less than $\kappa$ is denoted by $\mathcal{H}_{\kappa}$. The set of all infinite (respectively, infinite and regular) cardinals below $\kappa$ is denoted by $\operatorname{Card}(\kappa)$ (respectively, $\operatorname{Reg}(\kappa)$ ). The length of a finite sequence $\varrho$ is denoted by $\ell(\varrho)$. For a subset $S \subseteq \kappa$, we let $\operatorname{Tr}(S):=\left\{\alpha \in E_{>\omega}^{K} \mid S \cap \alpha\right.$ is stationary in $\left.\alpha\right\}$; we say that $S$ is nonreflecting (respectively, nonreflecting 
at inaccessibles) iff $\operatorname{Tr}(S)$ is empty (respectively, contains no inaccessible cardinals). For a set of ordinals $a$, we write $\operatorname{ssup}(a):=\sup \{\alpha+1 \mid \alpha \in a\}, \operatorname{acc}^{+}(a):=\{\alpha<\operatorname{ssup}(a) \mid \sup (a \cap \alpha)=\alpha>0\}$, $\operatorname{acc}(a):=a \cap \operatorname{acc}^{+}(a), \operatorname{nacc}(a):=a \backslash \operatorname{acc}(a)$, and $\operatorname{cl}(a):=a \cup \operatorname{acc}^{+}(a)$. For sets of ordinals, $a$ and $b$, we let $a \circledast b:=\{(\alpha, \beta) \in a \times b \mid \alpha<\beta\}$ and write $a<b$ to express that $a \times b$ coincides with $a \circledast b$.

For any set $\mathcal{A}$, we write $[\mathcal{A}]^{\chi}:=\{\mathcal{B} \subseteq \mathcal{A}|| \mathcal{B} \mid=\chi\}$ and $[\mathcal{A}]^{<\chi}:=\{\mathcal{B} \subseteq \mathcal{A}|| \mathcal{B} \mid<\chi\}$. This convention admits two refined exceptions:

- For an ordinal $\sigma$ and a set of ordinals $A$, we write $[A]^{\sigma}$ for $\{B \subseteq A \mid \operatorname{otp}(B)=\sigma\}$.

- For a set $\mathcal{A}$ that is either an ordinal or a collection of sets of ordinals, we interpret $[\mathcal{A}]^{2}$ as the collection of ordered pairs $\{(a, b) \in \mathcal{A} \times \mathcal{A} \mid a<b\}$.

In particular, $[\kappa]^{2}=\{(\alpha, \beta) \mid \alpha<\beta<\kappa\}$. Likewise, we identify $[\kappa]^{3}$ with $\{(\alpha, \beta, \gamma) \in \kappa \times \kappa \times \kappa \mid$ $\alpha<\beta<\gamma<\kappa\}$.

\section{Warming up}

\subsection{The foundations of walks on ordinals}

Definition 2.1 (folklore). $\kappa \rightarrow[\kappa]_{\theta}^{2}$ (respectively, $\kappa \rightarrow[\text { stat }]_{\theta}^{2}$ ) asserts the existence of a coloring $c:[\kappa]^{2} \rightarrow \theta$ such that for every cofinal (respectively, stationary) $X \subseteq \kappa$ and every $\tau \in \theta$, there exist $(x, y) \in[X]^{2}$ such that $c(x, y)=\tau$.

Likewise, $\kappa \nrightarrow[\kappa ; \kappa]_{\theta}^{2}$ (respectively, $\kappa \nrightarrow[\text { stat; stat }]_{\theta}^{2}$ ) asserts the existence of a coloring $c:[\kappa]^{2} \rightarrow$ $\theta$ such that for every two cofinal (respectively, stationary) $X, Y \subseteq \kappa$ and every $\tau \in \theta$, there exist $(x, y) \in X \circledast Y$ such that $c(x, y)=\tau$.

In an unpublished note from 1981, Todorcevic proved that $\omega_{1} \nrightarrow$ [stat; stat $]_{\omega_{1}}^{2}$ holds. A few years later, in [Tod87], the method of walks on ordinals was introduced, with the following theorem serving as the primary application.

Fact 2.2 (Todorcevic, [Tod87]). $\omega_{1} \rightarrow\left[\omega_{1}\right]_{\omega_{1}}^{2}$ holds. Furthermore, for every regular uncountable cardinal $\kappa$ admitting a nonreflecting stationary set, $\kappa \rightarrow[\kappa]_{\kappa}^{2}$ holds.

Later, by a series of results of Shelah concerning cardinals $\kappa>\boldsymbol{\aleph}_{1}$ together with a result of Moore concerning $\kappa=\boldsymbol{\aleph}_{1}, \kappa \rightarrow[\kappa ; \kappa]_{\kappa}^{2}$ holds for any cardinal $\kappa$ that is the successor of an infinite regular cardinal; see [RT13] for an historical account and a uniform proof of the following:

Fact 2.3 (Shelah, Moore). $v^{+} \nrightarrow\left[v^{+} ; v^{+}\right]_{v^{+}}^{2}$ holds for any infinite regular cardinal $v$.

In this subsection, we present a few basic components of the theory of walks on ordinals, which we will be using throughout the rest of the paper.

Definition 2.4. For a set of ordinals $\Gamma$, a $C$-sequence over $\Gamma$ is a sequence of sets $\left\langle C_{\alpha} \mid \alpha \in \Gamma\right\rangle$ such that for all $\alpha \in \Gamma, C_{\alpha}$ is a closed subset of $\alpha$ with $\sup \left(C_{\alpha}\right)=\sup (\alpha)$.

For the rest of this subsection, let us fix a $C$-sequence $\vec{C}=\left\langle C_{\alpha} \mid \alpha<\kappa\right\rangle$ over $\kappa$.

Definition 2.5 (Todorcevic, [Tod87]). From $\vec{C}$, we derive maps $\operatorname{Tr}:[\kappa]^{2} \rightarrow{ }^{\omega} \kappa, \rho_{2}:[\kappa]^{2} \rightarrow \omega$, $\operatorname{tr}:[\kappa]^{2} \rightarrow{ }^{<\omega} \kappa$ and $\lambda:[\kappa]^{2} \rightarrow \kappa$, as follows. Let $(\alpha, \beta) \in[\kappa]^{2}$ be arbitrary.

○ $\operatorname{Tr}(\alpha, \beta): \omega \rightarrow \kappa$ is defined by recursion on $n<\omega$ :

$$
\operatorname{Tr}(\alpha, \beta)(n):= \begin{cases}\beta, & n=0 \\ \min \left(C_{\operatorname{Tr}(\alpha, \beta)(n-1) \backslash \alpha),}\right. & n>0 \& \operatorname{Tr}(\alpha, \beta)(n-1)>\alpha \\ \alpha, & \text { otherwise }\end{cases}
$$

○ $\rho_{2}(\alpha, \beta):=\min \{n<\omega \mid \operatorname{Tr}(\alpha, \beta)(n)=\alpha\}$. 
$\circ \operatorname{tr}(\alpha, \beta):=\operatorname{Tr}(\alpha, \beta) \uparrow \rho_{2}(\alpha, \beta)$.

$\circ \lambda(\alpha, \beta):=\max \left\{\sup \left(C_{\operatorname{Tr}(\alpha, \beta)(i)} \cap \alpha\right) \mid i<\rho_{2}(\alpha, \beta)\right\}$.

The next two facts are quite elementary. They are reproduced with proofs as Claims 3.1.1 and 3.1.2 of [Rin14b].

Fact 2.6. Whenever $0<\beta<\gamma<\kappa$, if $\beta \notin \bigcup_{\alpha<\kappa} \operatorname{acc}\left(C_{\alpha}\right)$, then $\lambda(\beta, \gamma)<\beta$.

Fact 2.7. Whenever $\lambda(\beta, \gamma)<\alpha<\beta<\gamma<\kappa, \operatorname{tr}(\alpha, \gamma)=\operatorname{tr}(\beta, \gamma)^{-} \operatorname{tr}(\alpha, \beta)$.

Convention 2.8. For any coloring $f:[\kappa]^{2} \rightarrow \kappa$ and $\delta<\kappa$, while $(\delta, \delta) \notin[\kappa]^{2}$, we extend the definition of $f$ and agree to let $f(\delta, \delta):=0$.

Lemma 2.9. Let $(\alpha, \gamma) \in[\kappa]^{2}$. For every $\beta \in \operatorname{Im}(\operatorname{tr}(\alpha, \gamma))$,

$$
\lambda(\alpha, \gamma)=\max \{\lambda(\beta, \gamma), \lambda(\alpha, \beta)\} .
$$

Proof. Let $\beta$ be as above, so that $\operatorname{tr}(\alpha, \gamma)=\operatorname{tr}(\beta, \gamma)^{-} \operatorname{tr}(\alpha, \beta)$. We have

$$
\begin{aligned}
\lambda(\alpha, \gamma)= & \max \left\{\sup \left(C_{\tau} \cap \alpha\right) \mid \tau \in \operatorname{Im}(\operatorname{tr}(\alpha, \gamma))\right\}= \\
& \max \left\{\sup \left(C_{\tau_{0}} \cap \alpha\right), \sup \left(C_{\tau_{1}} \cap \alpha\right) \mid \tau_{0} \in \operatorname{Im}(\operatorname{tr}(\beta, \gamma)), \tau_{1} \in \operatorname{Im}(\operatorname{tr}(\alpha, \beta))\right\} \leq \\
& \max \left\{\sup \left(C_{\tau_{0}} \cap \beta\right), \sup \left(C_{\tau_{1}} \cap \alpha\right) \mid \tau_{0} \in \operatorname{Im}(\operatorname{tr}(\beta, \gamma)), \tau_{1} \in \operatorname{Im}(\operatorname{tr}(\alpha, \beta))\right\}= \\
& \max \{\lambda(\beta, \gamma), \lambda(\alpha, \beta)\},
\end{aligned}
$$

and

$$
\begin{aligned}
\lambda(\alpha, \gamma)= & \max \left\{\sup \left(C_{\tau_{0}} \cap \alpha\right), \sup \left(C_{\tau_{1}} \cap \alpha\right) \mid \tau_{0} \in \operatorname{Im}(\operatorname{tr}(\beta, \gamma)), \tau_{1} \in \operatorname{Im}(\operatorname{tr}(\alpha, \beta))\right\} \geq \\
& \max \left\{\sup \left(C_{\tau_{1}} \cap \alpha\right) \mid \tau_{1} \in \operatorname{Im}(\operatorname{tr}(\alpha, \beta))\right\}=\lambda(\alpha, \beta) .
\end{aligned}
$$

So, if $\lambda(\alpha, \gamma) \neq \max \{\lambda(\beta, \gamma), \lambda(\alpha, \beta)\}$, then $\lambda(\alpha, \gamma)<\lambda(\beta, \gamma)$, and we may fix the least $i<\rho_{2}(\beta, \gamma)$ to satisfy $\sup \left(C_{\operatorname{Tr}(\beta, \gamma)(i)} \cap \alpha\right)<\sup \left(C_{\operatorname{Tr}(\beta, \gamma)(i)} \cap \beta\right)$; but then $\operatorname{Tr}(\alpha, \gamma)(i+1)=\min \left(C_{\operatorname{Tr}(\beta, \gamma)(i)} \backslash \alpha\right)<$ $\beta \leq \operatorname{Tr}(\beta, \gamma)(i+1)$, contradicting the fact that $\operatorname{tr}(\beta, \gamma)^{-}\langle\beta\rangle \sqsubseteq \operatorname{tr}(\alpha, \gamma)$.

Definition 2.10. For every $(\alpha, \beta) \in[\kappa]^{2}$, we define an ordinal $\mathrm{\partial}_{\alpha, \beta} \in[\alpha, \beta]$ via:

$$
\mathrm{\partial}_{\alpha, \beta}:= \begin{cases}\alpha, & \text { if } \lambda(\alpha, \beta)<\alpha ; \\ \min (\operatorname{Im}(\operatorname{tr}(\alpha, \beta)), & \text { otherwise. }\end{cases}
$$

Lemma 2.11. Let $(\alpha, \beta) \in[\kappa]^{2}$ with $\alpha>0$. Then

1. $\lambda\left(\partial_{\alpha, \beta}, \beta\right)<\alpha .^{2}$

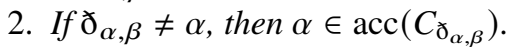

3. $\operatorname{tr}\left(\partial_{\alpha, \beta}, \beta\right) \sqsubseteq \operatorname{tr}(\alpha, \beta)$.

Proof. To avoid trivialities, assume that $\lambda(\alpha, \beta)=\alpha$. Let $\beta_{0}>\cdots>\beta_{n}>\beta_{n+1}$ denote the decreasing enumeration of the elements of $\operatorname{Im}(\operatorname{Tr}(\alpha, \beta))$, so that $\beta_{0}=\beta, \beta_{n}=\mathrm{\partial}_{\alpha, \beta}$, and $\beta_{n+1}=\alpha$. For each $i<n$, $C_{\beta_{i}} \cap\left[\alpha, \beta_{i+1}\right)$ is empty, so that $\min \left(C_{\beta_{i}} \backslash \beta_{n}\right)=\min \left(C_{\beta_{i}} \backslash \alpha\right)$ and $\sup \left(C_{\beta_{i}} \cap \beta_{n}\right)=\sup \left(C_{\beta_{i}} \cap \alpha\right)<\alpha$. Now, the three clauses follow immediately.

For the purpose of this paper, we also introduce the following ad hoc notation.

Definition 2.12. For every ordinal $\eta<\kappa$ and a pair $(\alpha, \beta) \in[\kappa]^{2}$, we let

$$
\eta_{\alpha, \beta}:=\min \left\{n<\omega \mid \eta \in C_{\operatorname{Tr}(\alpha, \beta)(n)} \text { or } n=\rho_{2}(\alpha, \beta)\right\}+1 .
$$




\subsection{Relationship to the C-sequence number}

Definition 2.13 (The $C$-sequence number of $\kappa$, [LHR21]). If $\kappa$ is weakly compact, then we define $\chi(\kappa):=0$. Otherwise, we let $\chi(\kappa)$ denote the least (finite or infinite) cardinal $\chi \leq \kappa$ such that for every $C$-sequence $\left\langle C_{\beta} \mid \beta<\kappa\right\rangle$, there exist $\Delta \in[\kappa]^{\kappa}$ and $b: \kappa \rightarrow[\kappa]^{\chi}$ with $\Delta \cap \alpha \subseteq \cup_{\beta \in b(\alpha)} C_{\beta}$ for every $\alpha<\kappa$.

Fact 2.14 (Todorcevic, [Tod07, Theorem 8.1.11]). If $\chi(\kappa)>1$, then $\kappa \nrightarrow[\kappa]_{\omega}^{2}$.

Fact 2.15 (Lambie-Hanson and Rinot, [LHR21]). If $\chi(\kappa) \leq 1$, then $\kappa$ is (in fact, greatly) Mahlo, and for every $C$-sequence $\left\langle C_{\beta} \mid \beta \in \operatorname{Reg}(\kappa)\right\rangle$ over $\operatorname{Reg}(\kappa)$, there exists a club $D \subseteq \kappa$ satisfying the following. For every $\alpha<\kappa$, there exists $\beta \in \operatorname{Reg}(\kappa)$, such that $D \cap \alpha \subseteq C_{\beta}$.

Lemma 2.16. If $\chi(\kappa) \leq 1$, then $\mathrm{P} \ell_{1}(\kappa, 1,2)$ fails.

Proof. Suppose that $\mathrm{P} \ell_{1}(\kappa, 1,2)$ holds.

Claim 2.16.1. There exists a function $s:[\kappa]^{2} \rightarrow \kappa$ satisfying the following:

1. For all $(\alpha, \beta) \in \kappa \circledast \operatorname{acc}(\kappa), \alpha<s(\alpha, \beta)<\beta$.

2. For every cofinal $A \subseteq \kappa, s^{\prime \prime}[A]^{2}$ is stationary.

Proof. Fix $\mathbf{t}:[\kappa]^{2} \rightarrow[\kappa]^{3}$ witnessing $\mathrm{P} \ell_{1}(\kappa, 1,2)$. Define $s:[\kappa]^{2} \rightarrow \kappa$ by letting $s(\alpha, \beta):=\beta^{*}$ whenever $\mathbf{t}(\alpha, \beta)=\left(\tau^{*}, \alpha^{*}, \beta^{*}\right)$ with $\alpha<\beta^{*}<\beta$, and letting $s(\alpha, \beta):=\alpha+1$, otherwise. To verify Clause (2), let $A$ be an arbitrary cofinal subset of $\kappa$. Set $C:=\operatorname{acc}^{+}(A)$ and $A^{\prime}:=A \backslash C$, so that $A^{\prime}$ is a discrete cofinal subset of $A$. As $\left\{\{\alpha\} \mid \alpha \in A^{\prime}\right\}$ is a subset of $[\kappa]^{1}$ consisting of $\kappa$ many pairwise disjoint sets, we may now fix a stationary $S \subseteq \kappa$ such that for all $\left(\alpha^{*}, \beta^{*}\right) \in[S]^{2}$, there exists $(\alpha, \beta) \in\left[A^{\prime}\right]^{2}$ with $\mathbf{t}(\alpha, \beta)=\left(0, \alpha^{*}, \beta^{*}\right)$. We claim that $s^{\prime \prime}[A]^{2}$ covers the stationary set $S^{*}:=(S \cap C) \backslash\{\min (S)\}$.

To see this, let $\beta^{*} \in S^{*}$ be arbitrary. Put $\alpha^{*}:=\min (S)$. Fix $(\alpha, \beta) \in\left[A^{\prime}\right]^{2}$ such that $\mathbf{t}(\alpha, \beta)=$ $\left(0, \alpha^{*}, \beta^{*}\right)$. We know that $\alpha^{*} \leq \alpha<\beta^{*} \leq \beta$ and that $\beta^{*} \in C$ while $\beta \in A \backslash C$. So $\alpha<\beta^{*}<\beta$, and hence $s(\alpha, \beta)=\beta^{*}$, as sought.

Suppose that $\chi(\kappa) \leq 1$, and yet there exists a function $s:[\kappa]^{2} \rightarrow \kappa$ as in the preceding claim. Set $C_{\omega}:=\omega$. For any uncountable $\beta \in \operatorname{Reg}(\kappa)$, let

$$
C_{\beta}:=\{\gamma<\beta \mid \forall \alpha<\gamma[s(\alpha, \beta)<\gamma]\}
$$

be the club of closure points of the function $s(\cdot, \beta)$. Note that, for any $\alpha<\beta, s(\alpha, \beta) \notin C_{\beta}$, since $\alpha<s(\alpha, \beta)$.

Now, by Fact 2.15, we may fix a club $D \subseteq \kappa$ with the property that, for every $\alpha<\kappa$, there exists $\beta \in \operatorname{Reg}(\kappa)$ with $D \cap \alpha \subseteq C_{\beta}$.

Recursively build a (discrete) subset $A \subseteq\left(\{0\} \cup\left(\operatorname{Reg}(\kappa) \backslash \omega_{1}\right)\right)$ such that for any nonzero $\beta \in A$, $\beta^{-}:=\sup (A \cap \beta)$ is smaller than $\beta$, and $D \cap\left(\beta^{-}+1\right) \subseteq C_{\beta}$. Then, let $E$ be the closure of $\bigcup\left\{C_{\beta} \backslash \beta^{-} \mid\right.$ $\beta \in A, \beta \neq 0\}$ in $\kappa$, and note that, for every $\beta \in A, E \cap\left(\beta^{-}, \beta\right)=C_{\beta} \cap\left(\beta^{-}, \beta\right)$.

As $A$ is cofinal, $S:=s^{\text {“ }}[A]^{2}$ is stationary, so that we may pick $\beta^{*} \in S \cap D \cap E$. Fix a pair $(\alpha, \beta) \in[A]^{2}$ with $s(\alpha, \beta)=\beta^{*}$.

Claim 2.16.2. $\beta^{*} \in C_{\beta}$.

Proof. As $(\alpha, \beta) \in[A]^{2}$, we we know that $\beta$ is a regular uncountable cardinal. So, by the hypothesis on $s, \alpha<\beta^{*}<\beta$. Now, there are two cases to consider:

- If $\beta^{*} \leq \beta^{-}$, then $\beta^{*} \in D \cap\left(\beta^{-}+1\right) \subseteq C_{\beta}$.

- Otherwise, $\beta^{-}<\beta^{*}<\beta$, so that $\beta^{*} \in E \cap\left(\beta^{-}, \beta\right)=C_{\beta} \cap\left(\beta^{-}, \beta\right)$.

However, we have observed earlier that $s(\alpha, \beta) \notin C_{\beta}$, meaning that $\beta^{*} \notin C_{\beta}$. This contradicts the preceding claim. 


\subsection{Relationship to Shelah's principle $\operatorname{Pr}_{1}$}

Definition 2.17 (Shelah, [She88]). $\operatorname{Pr}_{1}(\kappa, \kappa, \theta, \chi)$ asserts the existence of a coloring $c:[\kappa]^{2} \rightarrow \theta$ such that for every $\sigma<\chi$, every family $\mathcal{A} \subseteq[\kappa]^{\sigma}$ consisting of $\kappa$ many pairwise disjoint sets and every $i<\theta$, there is $(a, b) \in[\mathcal{A}]^{2}$ such that $c[a \times b]=\{i\}$.

Note that $\operatorname{Pr}_{1}(\kappa, \kappa, \theta, 2)$ is equivalent to $\kappa \nrightarrow[\kappa]_{\theta}^{2}$.

Lemma 2.18. Any of the following implies that $\operatorname{Pr}_{1}(\kappa, \kappa, \theta, \chi)$ holds:

1. $\mathrm{P} \ell_{1}(\kappa, \theta, \chi)$.

2. $\mathrm{P} \ell_{1}(\kappa, 1, \chi)$ and $\kappa \rightarrow[\operatorname{stat}(\kappa)]_{\theta}^{2}$.

3. $\mathrm{P} \ell_{1}(\kappa, \operatorname{cf}(\theta), \chi)$ and $\kappa \rightarrow[\operatorname{stat}(\kappa)]_{\eta}^{2}$ for all $\eta<\theta$.

4. $\mathrm{P} \ell_{1}(\kappa, v, \chi)$, and there exists a $\nu^{+}$-cc poset $\mathbb{P}$ such that $\mathbb{P}_{\mathbb{P}} \kappa \rightarrow[\kappa]_{\theta}^{2}$.

Proof.

(1) Let $\mathbf{t}:[\kappa]^{2} \rightarrow[\kappa]^{3}$ be a witness to $\mathrm{P} \ell_{1}(\kappa, \theta, \chi)$. Define $c^{*}:[\kappa]^{2} \rightarrow \theta$ via $c^{*}(\alpha, \beta):=\tau^{*}$ whenever $\mathbf{t}(\alpha, \beta)=\left(\tau^{*}, \alpha^{*}, \beta^{*}\right)$. Then $c^{*}$ witnesses $\operatorname{Pr}_{1}(\kappa, \kappa, \theta, \chi)$.

(2) Let $\mathbf{t}:[\kappa]^{2} \rightarrow[\kappa]^{3}$ be a witness to $\mathrm{P} \ell_{1}(\kappa, 1, \chi)$, and let $c:[\kappa]^{2} \rightarrow \theta$ be a witness to $\kappa \rightarrow[\operatorname{stat}(\kappa)]_{\theta}^{2}$. Define $c^{*}:[\kappa]^{2} \rightarrow \theta$ via $c^{*}(\alpha, \beta):=c\left(\alpha^{*}, \beta^{*}\right)$ whenever $\mathbf{t}(\alpha, \beta)=\left(\tau^{*}, \alpha^{*}, \beta^{*}\right)$. Then $c^{*}$ witnesses $\operatorname{Pr}_{1}(\kappa, \kappa, \theta, \chi)$.

(3) Let $\mathbf{t}:[\kappa]^{2} \rightarrow[\kappa]^{3}$ be a witness to $\mathrm{P} \ell_{1}(\kappa, \operatorname{cf}(\theta), \chi)$. By Clause (1), we may assume that $\theta$ is singular. Thus, let $\left\langle\eta_{i} \mid i<\operatorname{cf}(\theta)\right\rangle$ be an increasing sequence of cardinals, converging to $\theta$. For each $i<\operatorname{cf}(\theta)$, let $c_{i}:[\kappa]^{2} \rightarrow \eta_{i}$ be a witness to $\kappa \rightarrow[\operatorname{stat}(\kappa)]_{\eta_{i}}^{2}$. Define $c^{*}:[\kappa]^{2} \rightarrow \theta$ via $c^{*}(\alpha, \beta):=c_{i}\left(\alpha^{*}, \beta^{*}\right)$ whenever $\mathbf{t}(\alpha, \beta)=\left(i, \alpha^{*}, \beta^{*}\right)$. Then $c^{*}$ witnesses $\operatorname{Pr}_{1}(\kappa, \kappa, \theta, \chi)$.

(4) By Clause (1), we may assume that $v<\theta$. Let $\mathbf{t}:[\kappa]^{2} \rightarrow[\kappa]^{3}$ be a witness to $\mathrm{P} \ell_{1}(\kappa, v, \chi)$. Suppose that $\mathbb{P}$ is a $\nu^{+}$-cc poset such that $\mathbb{P}_{\mathbb{P}} \kappa \rightarrow[\kappa]_{\theta}^{2}$. Fix a $\mathbb{P}$-name $\dot{c}$ for a coloring witnessing $\kappa \rightarrow[\kappa]_{\theta}^{2}$ in the forcing extension by $\mathbb{P}$. Define $d:[\kappa]^{2} \rightarrow \mathcal{P}(\theta)$ via

$$
d(\alpha, \beta):=\left\{\tau<\theta \mid \exists p\left(p \mathbb{R}_{\mathbb{P}} \dot{c}(\check{\alpha}, \check{\beta})=\check{\tau}\right)\right\} .
$$

As $\mathbb{P}$ is $v^{+}$-cc, $|d(\alpha, \beta)| \leq v$ for every $(\alpha, \beta) \in[\kappa]^{2}$, so that we may define a function $e:[\kappa]^{3} \rightarrow \theta$ such that, all $(\alpha, \beta) \in[\kappa \backslash v]^{2}, d(\alpha, \beta) \subseteq\{e(i, \alpha, \beta) \mid i<\tau\}$. It follows that $e \circ \mathbf{t}$ witnesses $\operatorname{Pr}_{1}(\kappa, \kappa, \theta, \chi)$.

We now establish Theorem D.

Proposition 2.19. Suppose that $\kappa$ is weakly compact and $\chi \in \operatorname{Reg}(\kappa)$.

1. There exists a cofinality-preserving forcing extension in which $\kappa$ is strongly inaccessible, and there exists a coherent $\kappa$-Souslin tree, $\operatorname{Pr}_{1}(\kappa, \kappa, \kappa, \omega)$ holds, yet $\mathrm{P} \ell_{1}(\kappa)$ fails.

2. There exists a cofinality-preserving forcing extension in which $\kappa$ is strongly inaccessible, and there exists a nonreflecting stationary subset of $E_{\chi}^{\kappa}$, yet $\mathrm{P} \ell_{1}\left(\kappa, 1, \chi^{+}\right)$fails.

Proof.

(1) In [LHR21, §3], a cofinality-preserving forcing extension given by Kunen is revisited, in which $\kappa$ remains strongly inaccessible, and there exists a coherent $\kappa$-Souslin tree, so that $\operatorname{Pr}_{1}(\kappa, \kappa, \kappa, \omega)$ holds. It is shown there that $\chi(\kappa)=1$ holds in this model, so that, by Lemma $2.16, \mathrm{P} \ell_{1}(\kappa)$ fails.

(2) In [LHR21, §3], the authors give a cofinality-preserving forcing extension in which there exists a nonreflecting stationary subset of $E_{\chi}^{\kappa}$, and $\operatorname{Pr}_{1}\left(\kappa, \kappa, \kappa, \chi^{+}\right)$fails. By Fact 2.2 and Lemma 2.18, $\mathrm{P} \ell_{1}\left(\kappa, 1, \chi^{+}\right)$must fail in this model.

Next, we turn to deriving Theorem A: 
Corollary 2.20. Suppose that $\mathrm{P} \ell_{1}(\kappa)$ holds. For every cardinal $\theta \leq \kappa$, the following are equivalent:

1. $\kappa \rightarrow[\kappa]_{\theta}^{2}$.

2. $\kappa \rightarrow[\kappa ; \kappa]_{\theta}^{2}$.

3. $\operatorname{Pr}_{1}(\kappa, \kappa, \theta, \omega)$.

4. For every Abelian group $(G,+)$ of size $\kappa$, there exists a coloring $d: G \rightarrow \theta$ such that for all $X, Y \subseteq G$ of size $\kappa$ and every $\tau \in \theta$, there exist $x \in X$ and $y \in Y$ such that $d(x+y)=\tau$.

Proof. (3) $\Longrightarrow$ (2) $\Longrightarrow$ (1): This is trivial.

(1) $\Longrightarrow$ (3): By Lemma 2.18(2).

(3) $\Longrightarrow$ (4): By Lemma 3.4 and [FR17, Theorem 4.2].

$(4) \Longrightarrow(1)$ : As $\left([\kappa]^{<\omega}, \Delta\right)$ is an Abelian group of size $\kappa$, let us fix a coloring $d:[\kappa]^{<\omega} \rightarrow \theta$ as in Clause (4). Now define a coloring $c:[\kappa]^{2} \rightarrow \theta$ by stipulating $c(x, y):=d(\{x, y\})$. Clearly, $c$ witnesses that $\kappa \nrightarrow[\kappa]_{\theta}^{2}$ holds.

Remark 2.21. Compare the preceding with Conjecture 2 of [Rin14a].

Corollary 2.22. If $\mathrm{P} \ell_{1}(\kappa, 1, \chi)$ holds, then so does $\operatorname{Pr}_{1}(\kappa, \kappa, \omega, \chi)$.

Proof. To avoid trivialities, suppose that $\chi \geq 2$. Then, by Lemma 2.16, $\chi(\kappa)>1$. Finally, by Fact 2.14 and Theorem 2.18(2), $\operatorname{Pr}_{1}(\kappa, \kappa, \omega, \chi)$ holds.

We are now ready to derive Theorem B:

Corollary 2.23. Suppose that $\mathrm{P} \ell_{1}(\kappa)$ holds and $n$ is some positive integer. Then there exists a poset $\mathbb{P}$ such that $\mathbb{P}^{n}$ satisfies the $\kappa$-cc, but $\mathbb{P}^{n+1}$ does not.

Proof. By Corollary 2.22, in particular, we may fix a coloring $c:[\kappa]^{2} \rightarrow n+1$ witnessing $\operatorname{Pr}_{1}(\kappa, \kappa, n+$ $1, \omega)$. We define a poset $\mathbb{P}:=(P, \leq)$ by letting

$$
P:=\left\{(i, x) \mid i<n+1, x \in[\kappa]^{<\omega}, i \notin c^{“}[x]^{2}\right\},
$$

and letting $(i, x) \leq(j, y)$ iff $i=j$ and $x \supseteq y$. A moment's reflection makes it clear that $\{\langle(i,\{\alpha\})|$ $i<n+1\rangle|\alpha<\kappa\rangle$ forms a $\kappa$-sized antichain in $\mathbb{P}^{n+1}$.

We are left with showing that $\mathbb{P}^{n}$ does satisfy the $\kappa$-cc. To this end, let $A$ be an arbitrary $\kappa$-sized subset of $\mathbb{P}^{n}$. For every $p \in A$, write $p$ as $\left\langle\left(i_{j}^{p}, x_{j}^{p}\right) \mid j<n\right\rangle$. By the pigeonhole principle, we may assume the existence of a sequence $\left\langle i_{j} \mid j<n\right\rangle$ such that for every $p \in A,\left\langle i_{j}^{p} \mid j<n\right\rangle=\left\langle i_{j} \mid j<n\right\rangle$. Find $i^{*}<n+1$ such that $i^{*} \neq i_{j}$ for all $j<n$. By the $\Delta$-system lemma, we may also assume that, for every $j<n,\left\{x_{j}^{p} \mid\right.$ $p \in A\}$ forms a $\Delta$-system with some room $r_{j}$. Let $r:=\bigcup_{j<n} r_{j}$. Note that $r$ is finite (possibly empty). By further thinning out, we may assume that, for all $p \in A$ and $j<n, \min \left(x_{j}^{p} \backslash r_{j}\right)>\sup (r)$. By one last step of thinning out, we may finally secure that $\left\{\bigcup_{j<n} x_{j}^{p} \backslash r \mid p \in A\right\}$ form a family of $\kappa$-many pairwise disjoint finite sets.

Now, the choice of $c$ entails that we may find $p \neq q$ in $A$ such that:

1. $\max \left(\bigcup_{j<n} x_{j}^{p} \backslash r\right)<\min \left(\bigcup_{j<n} x_{j}^{q} \backslash r\right)$, and

2. $c\left[\left(\bigcup_{j<n} x_{j}^{p} \backslash r\right) \times\left(\bigcup_{j<n} x_{j}^{q} \backslash r\right)\right]=\left\{i^{*}\right\}$.

To see that $p$ and $q$ are compatible, fix arbitrary $j<n$ and $(\alpha, \beta) \in\left[x_{j}^{p} \cup x_{j}^{q}\right]^{2}$; we need to verify that $c(\alpha, \beta) \neq i_{j}$. There are three possible options:

จ If $(\alpha, \beta) \in\left[x_{j}^{p}\right]^{2} \cup\left[x_{j}^{q}\right]^{2}$, then since $i_{j}^{p}=i_{j}=i_{j}^{q}, c(\alpha, \beta) \neq i_{j}$.

- If $\alpha \in x_{j}^{p} \backslash x_{j}^{q}$ and $\beta \in x_{j}^{q} \backslash x_{j}^{p}$, then $\alpha \in x_{j}^{p} \backslash r_{j}$ and $\beta \in x_{j}^{q} \backslash r_{j}$, so that altogether $\alpha \in x_{j}^{p} \backslash r$ and $\beta \in x_{j}^{q} \backslash r$. by Clause (2), then, $c(\alpha, \beta)=i^{*}$. In particular, $c(\alpha, \beta) \neq i_{j}$.

- If $\alpha \in x_{j}^{q} \backslash x_{j}^{p}$ and $\beta \in x_{j}^{p} \backslash x_{j}^{q}$, then $\alpha \in x_{j}^{q} \backslash r$ and $\beta \in x_{j}^{p} \backslash r$, contradicting Clause (1). So this case does not exist. 


\section{Improved oscillation}

In [Rin14b], the first author introduced the following oscillation principle:

Definition 3.1. $\mathrm{P} \ell_{6}(\mu, v)$ asserts the existence of a map $d:{ }^{<\omega} \mu \rightarrow \omega$ such that for every sequence $\left\langle\left(u_{\alpha}, v_{\alpha}, \sigma_{\alpha}\right) \mid \alpha<\mu\right\rangle$ and a function $\varphi: \mu \rightarrow \mu$ satisfying:

1. $\varphi$ is eventually regressive. That is, $\varphi(\alpha)<\alpha$ for co-boundedly many $\alpha<\mu$;

2. $u_{\alpha}$ and $v_{\alpha}$ are nonempty elements of $\left[{ }^{<\omega} \mu\right]^{<v}$;

3. $\alpha \in \operatorname{Im}(\varrho)$ for all $\varrho \in u_{\alpha}$;

4. $\sigma_{\alpha} \frown\langle\alpha\rangle \sqsubseteq \sigma$ for all $\sigma \in v_{\alpha}$,

there exist $(\alpha, \beta) \in[\mu]^{2}$ with $\varphi(\alpha)=\varphi(\beta)$ such that for all $\varrho \in u_{\alpha}$ and $\sigma \in v_{\beta}, d(\varrho-\sigma)=\ell(\varrho)$.

The main result of $[\mathrm{Rin} 14 \mathrm{~b}, \S 2]$ states that $\mathrm{P} \ell_{6}\left(v^{+}, v\right)$ holds for every infinite regular cardinal $v$. In [RZ21], we show that $\mathrm{P} \ell_{6}\left(v^{+}, v\right)$ fails for every singular cardinal $v$ and that $\mathrm{P} \ell_{6}(\mu, \mu)$ fails for every infinite cardinal $\mu$.

In this paper, we shall be making use of two variations of $\mathrm{P} \ell_{6}\left(v^{+}, v\right)$. The first variation reads as follows:

Fact 3.2. Suppose that $\mu=v^{+}$for an infinite regular cardinal $v$. Then there exists a map $d:{ }^{<\omega} \mu \rightarrow$ $\omega \times \mu \times \mu \times \mu$ such that for every $\gamma^{*}<\mu$ and every sequence $\left\langle\left(u_{\alpha}, v_{\alpha}, \sigma_{\alpha}\right) \mid \alpha<\mu\right\rangle$ satisfying clauses (2)-(4) of Definition 3.1, there exist $(\alpha, \beta) \in[\mu]^{2}$ such that for all $\varrho \in u_{\alpha}$ and $\sigma \in v_{\beta}$, $d\left(\varrho^{-} \sigma\right)=\left(\ell(\varrho), \alpha, \beta, \gamma^{*}\right)$.

Proof. This follows immediately from Theorems 2.3 and 2.6 of [Rin14b].

The second variation reads as follows:

Lemma 3.3. Suppose that $\mu=v^{+}$for an infinite regular cardinal $v$. Then there exist a map $d_{0}:{ }^{<\omega} \mu \rightarrow$ $\omega$ and a $\mu$-additive normal ideal $J$ on $\mu$ with $E_{v}^{\mu} \notin J$ such that for every sequence $\left\langle\left(u_{\alpha}, v_{\alpha}, \sigma_{\alpha}\right)\right|$ $\alpha \in A\rangle$ with $A \in J^{+}$satisfying clauses (2)-(4) of Definition 3.1, there exist $(\alpha, \beta) \in[A]^{2}$ such that for all $\varrho \in u_{\alpha}$ and $\sigma \in v_{j}, d_{0}\left(\varrho^{-} \sigma\right)=\ell(\varrho)$.

Proof. By [Rin14b, Theorem 2.6], we may assume that $v^{<v}>v$. In particular, there exists a cardinal $\theta<v$ with $2^{\theta} \geq v$. So, by [She97, Claim 3.1] and [She97, Lemma 3.10], we may fix a $C$-sequence $\vec{C}=\left\langle C_{\beta} \mid \beta<\mu\right\rangle$ and a sequence of functions $\left\langle g_{\beta}: C_{\beta} \rightarrow \omega \mid \beta \in E_{v}^{\mu}\right\rangle$ such that:

$\circ \operatorname{otp}\left(C_{\beta}\right)=\operatorname{cf}(\beta)$ for all $\beta<\mu$.

○ For every club $D \subseteq \mu$, there exists some $\beta \in E_{v}^{\mu}$ such that for every $n<\omega, \sup \left\{\delta \in \operatorname{nacc}\left(C_{\beta}\right) \cap D \mid\right.$ $\left.g_{\beta}(\delta)=n\right\}=\beta$.

Fix a coloring $d:[\mu]^{2} \rightarrow \omega$ that satisfies $d(\alpha, \beta)=g_{\beta}\left(\min \left(C_{\beta} \backslash \alpha\right)\right)$ for all $\beta \in E_{v}^{\mu}$ and $\alpha<\beta$. Also, define a function $h:[\mu]^{<v} \rightarrow v$ via

$$
h(z):=\sup \left\{\operatorname{otp}\left(C_{\beta} \cap \alpha\right) \mid(\alpha, \beta) \in[z]^{2}\right\} .
$$

Next, define an ideal $J$ as follows: $A$ is in $J$ iff $A \subseteq \mu$, and there exists a club $D \subseteq \mu$ such that for every $\beta \in D \cap A \cap E_{v}^{\mu}$, there exists $n<\omega$ such that $\sup \left\{\delta \in \operatorname{nacc}\left(C_{\beta}\right) \cap D \mid g_{\beta}(\delta)=n\right\}<\beta$.

Claim 3.3.1. $J$ is a $\mu$-additive normal ideal on $\mu$ with $E_{v}^{\mu} \notin J$.

Proof. By the choice of $\vec{C}, E_{v}^{\mu} \notin J$. It is clear that for all $A \in J$ and $B \in[\mu]^{<\mu}, \mathcal{P}(A \cup B) \subseteq J$. Thus, it suffices to verify that $J$ is normal. So, suppose that $\left\langle A_{i} \mid i<\mu\right\rangle$ is a sequence of sets in $J$, and we shall prove that $A:=\nabla_{i<\mu} A_{i}$ is in $J$. For each $i<\mu$, fix a club $D_{i}$ witnessing that $A_{i} \in J$. We claim that $D:=\triangle_{i<\mu} D_{i}$ witnesses that $A$ is in $J$. Indeed, let $\beta \in D \cap A \cap E_{v}^{\mu}$ be arbitrary. Find $i<\beta$ such that $\beta \in A_{i}$. In particular, $\beta \in D_{i} \cap A_{i} \cap E_{\nu}^{\mu}$, and hence there exists $n<\omega$ such that $\sup \left\{\delta \in \operatorname{nacc}\left(C_{\beta}\right) \cap D \mid\right.$ $\left.g_{\beta}(\delta)=n\right\}<\beta$. 
The rest of the proof now follows that of [Rin14b, Theorem 2.3]. Given a sequence $\eta \in{ }^{<\omega} \mu$, let

$$
\mathcal{D}_{\eta}:=\{(i, j) \mid i<j<\ell(\eta) \& \eta(i)<\eta(j)\},
$$

and whenever $\mathcal{D}_{\eta} \neq \emptyset$, set

$\circ \mathrm{m}_{\eta}:=\max \left\{\operatorname{otp}\left(C_{\eta(j)} \cap \eta(i)\right) \mid(i, j) \in \mathcal{D}_{\eta}\right\}$

○ $\mathcal{P}_{\eta}:=\left\{(i, j) \in \mathcal{D}_{\eta} \mid \operatorname{otp}\left(C_{\eta(j)} \cap \eta(i)\right)=\mathfrak{m}_{\eta}\right\}$.

$\circ j_{\eta}:=\min \left\{j \mid \exists i(i, j) \in \mathcal{P}_{\eta}\right\}$.

$\circ \alpha_{\eta}:=\min \left\{\eta(i) \mid \exists j(i, j) \in \mathcal{P}_{\eta}\right\}$.

$\circ \beta_{\eta}:=\eta\left(j_{\eta}\right)$.

Finally, define $d_{0}:{ }^{<\omega} \mu \rightarrow \mu$ by letting for every $\eta \in{ }^{<\omega} \mu$ with $\mathcal{D}_{\eta} \neq \emptyset$ :

$$
d_{0}(\eta):=\max \left\{0, j_{\eta}-d\left(\alpha_{\eta}, \beta_{\eta}\right)\right\} .
$$

To verify this works, suppose that we are given a sequence $\left\langle\left(u_{\alpha}, v_{\alpha}, \sigma_{\alpha}\right) \mid \alpha \in A\right\rangle$ as in the statement of the lemma. Note that, without loss of generality, we may assume that $\alpha \notin \operatorname{Im}\left(\sigma_{\alpha}\right)$ for all $\alpha \in A$.

For every $\alpha \in A$, write $a_{\alpha}:=\bigcup\left\{\operatorname{Im}(\sigma) \mid \sigma \in u_{\alpha} \cup v_{\alpha}\right\}$, and $x_{\alpha}:=a_{\alpha} \backslash \alpha$. Let $\kappa$ be a large enough regular cardinal, and let $\unlhd_{K}$ be a well-ordering of $\mathcal{H}_{\kappa}$. Let $\left\langle M_{\delta} \mid \delta<\mu\right\rangle$ be a continuous $\in$-chain of elementary submodels of $\left(\mathcal{H}_{\kappa}, \in, \unlhd_{K}\right)$, each of size $v$, such that $v \subseteq M_{0}$ and $\left\{h,\left\langle a_{\alpha} \mid \alpha \in A\right\rangle\right\} \in M_{0}$.

Write $D:=\left\{\delta<\mu \mid M_{\delta} \cap \mu=\delta\right\}$. As $A \in J^{+}$, let us pick $\beta \in D \cap A \cap E_{v}^{\mu}$ such that $\sup \{\delta \in$ $\left.\operatorname{nacc}\left(C_{\beta}\right) \cap D \mid g_{\beta}(\delta)=n\right\}=\beta$ for all $n<\omega$. Put $\xi:=\sup \left(a_{\beta} \cap \beta\right)+1$. As $\left|a_{\beta}\right|<\operatorname{cf}(\beta), \xi<\beta$. Let $f: v \rightarrow \xi$ be the $\unlhd_{K}$-least surjection. From $\left|a_{\beta}\right|<v$ and regularity of $v$, let $i^{\prime}<v$ be large enough such that $a_{\beta} \cap \beta \subseteq f\left[i^{\prime}\right]$. Write $n^{*}:=\ell\left(\sigma_{\beta}\right), z:=f\left[i^{\prime}\right], \epsilon:=h\left(a_{\beta} \cup z\right)$, and

$$
A^{\prime}:=\left\{\alpha \in A \mid a_{\alpha} \cap \alpha \subseteq z, h\left(a_{\alpha} \cup z\right)=\epsilon\right\} .
$$

Pick $\delta \in \operatorname{nacc}\left(C_{\beta}\right) \cap D$ above $\xi$ with otp $\left(C_{\beta} \cap \delta\right)>\epsilon$ such that $g_{\beta}(\delta)=n^{*}$. As $\xi \in M_{\delta}, A^{\prime} \in M_{\delta}$. Since $\beta \in A^{\prime} \backslash M_{\delta}$, $\sup \left(A^{\prime} \cap M_{\delta}\right)=\delta$. So, let us pick $\alpha \in A^{\prime} \cap M_{\delta}$ above $\max \left(C_{\beta} \cap \delta\right)$.

\section{Claim 3.3.2.}

1. $h\left(a_{\alpha} \cup z\right)=\epsilon$.

2. $a_{\alpha} \cap \alpha \subseteq z$.

3. $x_{\alpha} \subseteq\left(\max \left(C_{\beta} \cap \delta\right), \delta\right)$. In particular, otp $\left(C_{\beta} \cap \alpha\right)>\epsilon$.

4. $d(\alpha, \beta)=\ell\left(\sigma_{\beta}\right)$.

Proof. By the same proof of [Rin14b, Claim 2.3.1].

To see that the pair $(\alpha, \beta)$ is as sought, suppose that we are given $\varrho \in u_{\alpha}$ and $\sigma \in v_{\beta}$, and let us show that $d_{0}(\eta)=\ell(\varrho)$ for $\eta:=\varrho \frown \sigma$.

As $\alpha \in \operatorname{Im}(\varrho)$ and $\beta \in \operatorname{Im}(\sigma)$, there exist $\hat{i}<\hat{j}<\ell(\eta)$ such that $\eta(\hat{i})=\alpha$ and $\eta(\hat{j})=\beta$. So $(\hat{i}, \hat{j})$ witnesses that $\mathcal{D}_{\eta} \neq \emptyset$, and then, by Claim 3.3.2(3), we have $\mathfrak{m}_{\eta} \geq \operatorname{otp}\left(C_{\beta} \cap \alpha\right)>\epsilon$.

Claim 3.3.3. For every $(i, j) \in \mathcal{P}_{\eta}$ :

1. $\{\eta(i), \eta(j)\} \nsubseteq\left(a_{\alpha} \cup z\right)$, and $\{\eta(i), \eta(j)\} \nsubseteq\left(a_{\beta} \cup z\right)$.

2. If $\eta(i) \in a_{\alpha}$, then $\eta(j) \notin a_{\beta} \cap \beta$.

3. $\varrho \sqsubseteq \eta \uparrow j$.

4. $\eta(j)=\beta$.

5. $\eta(i) \in x_{\alpha}$.

Proof. By the same proof of [Rin14b, Claim 2.3.2].

As $\beta \notin \operatorname{Im}\left(\sigma_{\beta}\right)$, we get from the minimality of $j_{\eta}$ together with Clauses (3) and (4) of the preceding claim that

$$
\eta \uparrow\left(j_{\eta}+1\right)=\varrho \frown \sigma_{\beta} \frown\langle\beta\rangle .
$$


So $\beta_{\eta}=\beta$ and $j_{\eta}=\ell\left(\varrho-\sigma_{\beta}\right)$. By Clause (5) of the preceding claim, $\alpha_{\eta} \in x_{\alpha}$. Then, by Claim 3.3.2(3), we get that $\operatorname{otp}\left(C_{\beta} \cap \alpha_{\eta}\right)=\operatorname{otp}\left(C_{\beta} \cap \alpha\right)$. Recalling that $\min \left(x_{\alpha}\right)=\alpha \in \operatorname{Im}(\varrho)$, Claims 3.3.3(5) and 3.3.2(3) then imply that $\alpha_{\eta}=\alpha$.

Recalling Claim 3.3.2(4), we altogether infer that

$$
\eta \uparrow\left(j_{\eta}-d\left(\alpha_{\eta}, \beta_{\eta}\right)\right)=(\varrho \frown \sigma) \uparrow\left(\ell\left(\varrho \frown \sigma_{\beta}\right)-\ell\left(\sigma_{\beta}\right)\right)=\varrho .
$$

So, $d_{0}(\sigma)=\max \left\{0, j_{\eta}-d\left(\alpha_{\eta}, \beta_{\eta}\right)\right\}=\ell(\varrho)$, as sought.

\section{Clause (2) of Theorem $C$}

In this section, we suppose that $\chi \in \operatorname{Reg}(\kappa)$ is a cardinal satisfying $\chi^{+}<\kappa$, and there exists a stationary subset of $E_{\geq \chi}^{\kappa}$ that does not reflect. We shall construct a witness to $\mathrm{P} \ell_{1}(\kappa, \kappa, \chi)$. The proof is split into two cases: $\chi^{++}<\kappa$ and $\chi^{++}=\kappa$.

\subsection{Case I}

In this subsection, we suppose that $\chi^{++}<\kappa$. Note that, by Proposition 2.19(2), the result of this subsection cannot be improved.

Lemma 4.1. There exists $v \in \operatorname{Reg}(\kappa) \backslash \chi$ with $v^{+}<\kappa$ and a stationary subset $\Gamma \subseteq E_{\geq \chi}^{\kappa} \cap E_{\neq v^{+}}^{\kappa}$ that does not reflect.

Proof. By the hypothesis of this section, let us fix a stationary subset $R \subseteq E_{\geq \chi}^{\kappa}$ that does not reflect.

If $R \cap \operatorname{Reg}(\kappa)$ is stationary, then we may simply let $v:=\chi$ and $\Gamma:=R \cap \operatorname{Reg}(\kappa) \backslash\left(\nu^{+}+1\right)$. Next, suppose that $R \cap \operatorname{Reg}(\kappa)$ is nonstationary, and use Fodor's lemma to fix a regular cardinal $\theta \geq \chi$ for which $R \cap E_{\theta}^{\kappa}$ is stationary.

- If $\theta^{+}<\kappa$, then we let $v:=\theta$. It follows that $v^{+}<\kappa$, and $E_{\theta}^{\kappa} \cap E_{\nu^{+}}^{\kappa}=\emptyset$, so that $\Gamma:=R \backslash E_{v^{+}}^{\kappa}$ is as sought.

- If $\theta^{+}=\kappa$, then we let $v:=\chi$. As $\chi^{++}<\kappa$, we infer that $v^{+}<\theta<\kappa$, so that $E_{\theta}^{\kappa} \cap E_{v^{+}}^{\kappa}=\emptyset$ and $\Gamma:=R \backslash E_{v^{+}}^{\kappa}$ is as sought.

Let $v$ and $\Gamma$ be given by the preceding lemma. Set $\mu:=v^{+}$, so that $\Gamma \cap E_{\mu}^{\kappa}=\emptyset$. Fix a surjection $g: \kappa \rightarrow \kappa \times \kappa$ such that $G_{\eta, \tau}:=\{\delta \in \Gamma \mid g(\delta)=(\eta, \tau)\}$ is stationary for every $(\eta, \tau) \in \kappa \times \kappa$. Fix another surjection $h: \kappa \rightarrow \mu$ such that $H_{i}:=\{\alpha \in \Gamma \mid h(\alpha)=i\}$ is stationary for every $i<\mu$.

As $\Gamma$ is nonreflecting, let $\vec{C}=\left\langle C_{\alpha} \mid \alpha<\kappa\right\rangle$ be a sequence such that $C_{\alpha+1}=\{\alpha\}$ for every $\alpha<\kappa$, and such that for every $\alpha \in \operatorname{acc}(\kappa), C_{\alpha}$ is a club in $\alpha$ with $\operatorname{acc}\left(C_{\alpha}\right) \cap \Gamma=\emptyset$. By a club-guessing theorem due to Shelah (see also [BR19, Remark 1.5 and Lemma 2.5]), we may also assume that, for every club $D \subseteq \kappa$, there exists $\gamma \in \Gamma$ with $\sup \left(\operatorname{nacc}\left(C_{\gamma}\right) \cap D\right)=\gamma$. Recalling Subsection 2.1, we now let $\operatorname{Tr}, \operatorname{tr}, \lambda$ and $\rho_{2}$ be the characteristic functions of walking along $\vec{C}$, and let $\eta_{\alpha, \beta}$ be the notation established in Definition 2.12. In addition, we consider yet another function $\operatorname{tr}_{h}:[\kappa]^{2} \rightarrow{ }^{<\omega} \mu$ that is defined via $\operatorname{tr}_{h}(\alpha, \beta):=h \circ \operatorname{tr}(\alpha, \beta)$.

Appeal to Lemma 3.3 to fix a map $d_{0}:{ }^{<\omega} \mu \rightarrow \omega$ and its corresponding $\mu$-additive proper ideal $J$. Define $c:[\kappa]^{2} \rightarrow \kappa \times \kappa$ via

$$
c(\alpha, \beta):=g\left(\operatorname{Tr}(\alpha, \beta)\left(d_{0}\left(\operatorname{tr}_{h}(\alpha, \beta)\right)\right)\right) .
$$

We are finally ready to define our transformation.

Definition 4.2. Define $\mathbf{t}:[\kappa]^{2} \rightarrow[\kappa]^{3}$ by letting, for all $(\alpha, \beta) \in[\kappa]^{2}, \mathbf{t}(\alpha, \beta):=\left(\tau, \alpha^{*}, \beta^{*}\right)$ provided that the following conditions are met:

$\circ(\eta, \tau):=c(\alpha, \beta)$ and $\max \{\eta+1, \tau\}<\alpha$,

○ $\beta^{*}=\operatorname{Tr}(\alpha, \beta)\left(\eta_{\alpha, \beta}\right)$ is $>\alpha$, and

$\circ \alpha^{*}=\operatorname{Tr}(\eta+1, \alpha)\left(\eta_{\eta+1, \alpha}\right)$. 
Otherwise, let $\mathbf{t}(\alpha, \beta):=(0, \alpha, \beta)$.

To verify that $\mathbf{t}$ witnesses $\mathrm{P} \ell_{1}(\kappa, \kappa, \chi)$, suppose that we are given a family $\mathcal{A} \subseteq[\kappa]^{<\chi}$ consisting of $\kappa$ many pairwise disjoint sets. Fix a sequence $\vec{x}=\left\langle x_{\delta} \mid \delta<\kappa\right\rangle$ such that for all $\delta<\kappa, x_{\delta} \in \mathcal{A}$ with $\min \left(x_{\delta}\right)>\delta$.

Definition 4.3. For $\eta<\kappa, S_{\eta}$ denotes the set of all $\epsilon<\kappa$ with the property that, for every $\varsigma<\kappa$, there exist $I \in J^{+}$and a sequence $\left\langle\beta_{i} \mid i \in I\right\rangle \in \prod_{i \in I} H_{i} \backslash \varsigma$, such that for all $i \in I$ and $\beta \in x_{\beta_{i}}$ :

(i) $i \in \operatorname{Im}\left(\operatorname{tr}_{h}(\epsilon, \beta)\right)$.

(ii) $\lambda(\epsilon, \beta)=\eta$.

(iii) $\rho_{2}(\epsilon, \beta)=\eta_{\epsilon, \beta}$.

Lemma 4.4. There exists $\eta<\kappa$ for which $S_{\eta}$ is stationary.

Proof. By the pressing down lemma, it suffices to prove that, for every club $D \subseteq \kappa$, there exist $\epsilon \in D$ and $\eta<\epsilon$ for which $\epsilon \in S_{\eta}$. Thus, let $D$ be an arbitrary club in $\kappa$.

Define a function $f: \Gamma \rightarrow \kappa$ via

$$
f(\delta):=\sup \left\{\lambda(\delta, \beta) \mid \beta \in x_{\delta}\right\}
$$

By Fact 2.6 and since $\left|x_{\delta}\right|<\chi \leq \operatorname{cf}(\delta)$ for all $\delta \in \Gamma, f$ is regressive. So, for all $i<\mu$, let us pick a stationary subset $\bar{H}_{i} \subseteq H_{i}$ such that $f \uparrow \bar{H}_{i}$ is constant. Set $\zeta:=\sup \left(f\left[\bigcup_{i<\mu} \bar{H}_{i}\right]\right)$. Now, by the club-guessing feature of $\vec{C}$, let us pick $\gamma \in \Gamma$ with $\sup \left(\operatorname{nacc}\left(C_{\gamma}\right) \cap(D \backslash \zeta)\right)=\gamma$.

Let $\varsigma<\kappa$. Fix a sequence $\left\langle\beta_{i}^{\varsigma} \mid i<\mu\right\rangle \in \prod_{i<\mu} \bar{H}_{i} \backslash \max \{\gamma+1, \varsigma\}$. For every $i<\mu$, by Fact 2.6, $\lambda\left(\gamma, \beta_{i}^{S}\right)<\gamma$, so as $\gamma \in \Gamma \subseteq E_{\neq \mu}^{\kappa}$ and as $J$ is a $\mu$-additive proper ideal on $\mu$, we may fix $I^{S} \in J^{+}$ along with some ordinal $\xi^{\varsigma}<\gamma$ such that $\lambda\left(\gamma, \beta_{i}^{S}\right) \leq \xi^{\varsigma}$ for all $i \in I^{\varsigma}$. Then, pick a large enough $\epsilon^{S} \in \operatorname{nacc}\left(C_{\gamma}\right) \cap D$ such that $\sup \left(C_{\gamma} \cap \epsilon^{S}\right)>\max \left\{\xi^{S}, \zeta\right\}$.

Next, by the pigeonhole principle, let us fix $\epsilon \in \operatorname{nacc}\left(C_{\gamma}\right) \cap D$ for which $\Sigma:=\left\{\varsigma<\kappa \mid \epsilon^{\varsigma}=\epsilon\right\}$ is cofinal in $\kappa$. Put $\eta:=\sup \left(C_{\gamma} \cap \epsilon\right)$, so that $\eta<\epsilon$.

We already know that $\epsilon \in D$. To see that $\epsilon \in S_{\eta}$, let $\varsigma<\kappa$ be arbitrary. By increasing $\varsigma$, we may assume that $\varsigma \in \Sigma$. Let $i \in I^{\varsigma}$ and $\beta \in x_{\beta_{i}^{\varsigma}}$ be arbitrary. As $\beta_{i}^{\varsigma} \in H_{i}$, it suffices to show that:

(i') $\operatorname{tr}(\epsilon, \beta)=\operatorname{tr}\left(\beta_{i}^{S}, \beta\right)^{-} \operatorname{tr}\left(\epsilon, \beta_{i}^{S}\right)$.

(ii') $\lambda(\epsilon, \beta)=\eta$.

(iii') $\rho_{2}(\epsilon, \beta)=\eta_{\epsilon, \beta}$.

We have:

$$
\lambda\left(\beta_{i}^{\varsigma}, \beta\right) \leq f\left(\beta_{i}^{\varsigma}\right) \leq \zeta \leq \max \left\{\lambda\left(\gamma, \beta_{i}^{\varsigma}\right), \zeta\right\} \leq \max \left\{\xi^{\varsigma}, \zeta\right\}<\eta<\epsilon<\gamma<\beta_{i}^{\varsigma}<\beta
$$

It thus follows from Fact 2.7 that Clause (i') is satisfied. It also follows from Fact 2.7 that $\operatorname{tr}\left(\epsilon, \beta_{i}^{S}\right)=$ $\operatorname{tr}\left(\gamma, \beta_{i}^{S}\right)^{-} \operatorname{tr}(\epsilon, \gamma)$, so that altogether

$$
\operatorname{tr}(\epsilon, \beta)=\operatorname{tr}\left(\beta_{i}^{\varsigma}, \beta\right)^{-} \operatorname{tr}\left(\gamma, \beta_{i}^{\varsigma}\right)^{-} \operatorname{tr}(\epsilon, \gamma) .
$$

By Lemma 2.9 and the above equation,

$$
\lambda(\epsilon, \beta)=\max \left\{\lambda\left(\beta_{i}^{\varsigma}, \beta\right), \lambda\left(\gamma, \beta_{i}^{\varsigma}\right), \lambda(\epsilon, \gamma)\right\}
$$

Recall that $\max \left\{\lambda\left(\beta_{i}^{S}, \beta\right), \lambda\left(\gamma, \beta_{i}^{S}\right)\right\} \leq \max \left\{\zeta, \xi^{S}\right\}<\eta$. As $\epsilon \in C_{\gamma}$, we infer that $\lambda(\epsilon, \gamma)=$ $\sup \left(C_{\gamma} \cap \epsilon\right)=\eta$. In effect, $\lambda(\epsilon, \beta)=\eta$ and $\rho_{2}(\epsilon, \beta)=\eta_{\epsilon, \beta}$. 
Let $\eta$ be given by the preceding lemma. Let $D$ be a club in $\kappa$ such that for all $\delta \in D$, there exists $M_{\delta} \prec \mathcal{H}_{\kappa^{+}}$containing the parameter $p:=\left\{\Gamma, S_{\eta}, \vec{x}, \vec{C}, h, \mu\right\}$ and satisfying $M_{\delta} \cap \kappa=\delta$. Finally, let

$$
S^{*}:=S_{\eta} \cap \triangle_{\tau<K} \operatorname{acc}^{+}\left(G_{\eta, \tau} \cap \bigcap_{j<\mu} \operatorname{acc}^{+}\left(H_{j} \cap D\right)\right) .
$$

Lemma 4.5. Let $\left(\tau^{*}, \alpha^{*}, \beta^{*}\right) \in \kappa \circledast S^{*} \circledast S^{*}$. There exists $(a, b) \in[\mathcal{A}]^{2}$ such that $\mathbf{t}[a \times b]=\left\{\left(\tau^{*}, \alpha^{*}, \beta^{*}\right)\right\}$.

Proof. As $\beta^{*} \in S^{*} \subseteq S_{\eta}$, let us pick $I \in J^{+}$and a sequence $\left\langle\beta_{i} \mid i \in I\right\rangle \in \prod_{i \in I} H_{i} \backslash\left(\beta^{*}+1\right)$ such that for all $i \in I$ and $\beta \in x_{\beta_{i}}$ :

1. $i \in \operatorname{Im}\left(\operatorname{tr}_{h}\left(\beta^{*}, \beta\right)\right)$.

2. $\lambda\left(\beta^{*}, \beta\right)=\eta$.

3. $\rho_{2}\left(\beta^{*}, \beta\right)=\eta_{\beta^{*}, \beta}$.

As $\left(\tau^{*}, \beta^{*}\right) \in \kappa \circledast S^{*}$, pick a large enough $\varepsilon \in\left(G_{\eta, \tau^{*}} \cap \bigcap_{j<\mu} \operatorname{acc}^{+}\left(H_{j} \cap D\right)\right) \cap \beta^{*}$ such that $\sup \left(C_{\beta^{*}} \cap \varepsilon\right)>\alpha^{*}$. In particular, $\lambda\left(\varepsilon, \beta^{*}\right)>\alpha^{*}>\eta$.

For all $j<\mu$, as $\varepsilon \in \Gamma \cap \operatorname{acc}^{+}\left(H_{j} \cap D\right)$, Fact 2.6 entails that we may pick a large enough $\delta_{j} \in H_{j} \cap D \cap \varepsilon$ such that $\delta_{j}>\lambda\left(\varepsilon, \beta^{*}\right)$. As $M_{\delta_{j}}$ contains $p$, we have that $S_{\eta} \in M_{\delta_{j}}$. As $\delta_{j} \in \Gamma$, Fact 2.6 entails that $\varsigma_{j}:=\max \left\{\alpha^{*}, \lambda\left(\varepsilon, \beta^{*}\right), \lambda\left(\delta_{j}, \varepsilon\right)\right\}+1$ is smaller than $\delta_{j}$. Since $\alpha^{*} \in M_{\delta_{j}} \cap S_{\eta}$, we may then find $\alpha_{j} \in M_{\delta_{j}} \cap\left(\bigcup_{i<\mu} H_{i}\right) \backslash \varsigma_{j}$ such that for all $\alpha \in x_{\alpha_{j}}$ :

(2') $\lambda\left(\alpha^{*}, \alpha\right)=\eta$.

(3') $\rho_{2}\left(\alpha^{*}, \alpha\right)=\eta_{\alpha^{*}, \alpha}$.

Note that from $\alpha_{j} \in M_{\delta_{j}}$, it follows that $\sup \left(x_{\alpha_{j}}\right)<\delta_{j}$. Write $a_{j}:=x_{\alpha_{j}}$ and $b_{i}:=x_{\beta_{i}}$. Let $(i, j, \alpha, \beta) \in I \times \mu \times a_{j} \times b_{i}$ be arbitrary. Then:

$$
\eta<\eta+1<\alpha^{*}<\varsigma_{j} \leq \alpha_{j}<\alpha<\delta_{j}<\varepsilon<\beta^{*}<\beta_{i}<\beta .
$$

In particular, Fact 2.7 yields the following conclusions:

(a) From $\lambda\left(\beta^{*}, \beta\right)=\eta<\alpha<\beta^{*}<\beta$, we have $\operatorname{tr}(\alpha, \beta)=\operatorname{tr}\left(\beta^{*}, \beta\right)^{-} \operatorname{tr}\left(\alpha, \beta^{*}\right)$.

(b) From $\lambda\left(\varepsilon, \beta^{*}\right)<\varsigma_{j}<\alpha<\varepsilon<\beta^{*}$, we have $\operatorname{tr}\left(\alpha, \beta^{*}\right)=\operatorname{tr}\left(\varepsilon, \beta^{*}\right)^{-} \operatorname{tr}(\alpha, \varepsilon)$.

(c) From $\lambda\left(\delta_{j}, \varepsilon\right)<\varsigma_{j}<\alpha<\delta_{j}<\varepsilon$, we have $\operatorname{tr}(\alpha, \varepsilon)=\operatorname{tr}\left(\delta_{j}, \varepsilon\right)^{-} \operatorname{tr}\left(\alpha, \delta_{j}\right)$.

So that, altogether,

$$
\operatorname{tr}(\alpha, \beta)=\operatorname{tr}\left(\beta^{*}, \beta\right)^{-} \operatorname{tr}\left(\varepsilon, \beta^{*}\right)^{-} \operatorname{tr}\left(\delta_{j}, \varepsilon\right)^{-} \operatorname{tr}\left(\alpha, \delta_{j}\right) .
$$

In addition, from $\lambda\left(\alpha^{*}, \alpha\right)=\eta<\eta+1<\alpha^{*}<\alpha$, we infer that

(d) $\operatorname{tr}(\eta+1, \alpha)=\operatorname{tr}\left(\alpha^{*}, \alpha\right)^{-} \operatorname{tr}\left(\eta+1, \alpha^{*}\right)$.

For each $i \in I$, denote $u_{i}:=\left\{\operatorname{tr}_{h}(\varepsilon, \beta) \mid \beta \in b_{i}\right\}$. For each $j<\mu$, denote $v_{j}:=\left\{\operatorname{tr}_{h}(\alpha, \varepsilon) \mid \alpha \in a_{j}\right\}$.

\section{Claim 4.5.1.}

(i) For every $i \in I, i \in \operatorname{Im}(\varrho)$ for all $\varrho \in u_{i}$.

(ii) For every $j<\mu$, there exists $\sigma_{j} \in{ }^{<\omega} \mu$ such that $\sigma_{j}{ }^{-}\langle j\rangle \sqsubseteq \sigma$ for all $\sigma \in v_{j}$.

Proof. (i) For all $\beta \in b_{i}, \operatorname{tr}_{h}(\varepsilon, \beta)=\operatorname{tr}_{h}\left(\beta^{*}, \beta\right)^{-} \operatorname{tr}_{h}\left(\varepsilon, \beta^{*}\right)$, so the conclusion follows from Clause (1). (ii) Since $\delta_{j} \in H_{j}$, by Clause (c) above, $\operatorname{tr}_{h}\left(\delta_{j}, \varepsilon\right)^{-}\langle j\rangle \sqsubseteq \sigma$ for all $\sigma \in v_{j}$.

Next, by the choice of $d_{0}$, fix $(i, j) \in[I]^{2}$ such that $d_{0}\left(\varrho^{-} \sigma\right)=\ell(\varrho)$ for all $\varrho \in u_{i}$ and $\sigma \in v_{j}$. Set $a:=a_{j}$ and $b:=b_{i}$, so that $(a, b) \in[\mathcal{A}]^{2}$.

To see that $\mathbf{t}[a \times b]=\left\{\left(\tau^{*}, \alpha^{*}, \beta^{*}\right)\right\}$, fix arbitrary $\alpha \in a$ and $\beta \in b$. 
Claim 4.5.2. $c(\alpha, \beta)=\left(\eta, \tau^{*}\right)$.

Proof. Write $\varrho:=\operatorname{tr}_{h}(\varepsilon, \beta)$ and $\sigma:=\operatorname{tr}_{h}(\alpha, \varepsilon)$. Then:

$\circ \operatorname{tr}_{h}(\alpha, \beta)=\varrho^{-} \sigma$.

○ $d_{0}\left(\operatorname{tr}_{h}(\alpha, \beta)\right)=\ell(\varrho)=\ell(\operatorname{tr}(\varepsilon, \beta))=\rho_{2}(\varepsilon, \beta)$.

○ $\operatorname{Tr}(\alpha, \beta)\left(d_{0}\left(\operatorname{tr}_{h}(\alpha, \beta)\right)\right)=\operatorname{Tr}(\alpha, \beta)\left(\rho_{2}(\varepsilon, \beta)\right)=\varepsilon$.

So, $c(\alpha, \beta)=g\left(\operatorname{Tr}(\alpha, \beta)\left(d_{0}\left(\operatorname{tr}_{h}(\alpha, \beta)\right)\right)\right)=g(\varepsilon)=\left(\eta, \tau^{*}\right)$.

By Clause (a) above, $\operatorname{tr}(\alpha, \beta)=\operatorname{tr}\left(\beta^{*}, \beta\right)^{-} \operatorname{tr}\left(\alpha, \beta^{*}\right)$, so Clause (3) above implies that $\eta_{\alpha, \beta}=\eta_{\beta^{*}, \beta}=$ $\rho_{2}\left(\beta^{*}, \beta\right)$.

By Clause (d) above, $\operatorname{tr}(\eta+1, \alpha)=\operatorname{tr}\left(\alpha^{*}, \alpha\right)^{-} \operatorname{tr}\left(\eta+1, \alpha^{*}\right)$, so Clause (3') above implies that $\eta_{\eta+1, \alpha}=\eta_{\alpha^{*}, \alpha}=\rho_{2}\left(\alpha^{*}, \alpha\right)$. Altogether, $\mathrm{t}(\alpha, \beta)=\left(\tau^{*}, \alpha^{*}, \beta^{*}\right)$.

\subsection{Case II}

In this subsection, we suppose that $\chi^{++}=\kappa$. Denote $\mu:=\chi^{+}$. It is clear that $\mathrm{P} \ell_{1}(\kappa, \kappa, \chi)$ is equivalent to $\mathrm{P} \ell_{1}(\kappa, \mu, \chi)$, so we shall focus on constructing a witness to the latter. Denote $\Gamma:=E_{\mu}^{\kappa}$.

Fix a function $h: \kappa \rightarrow \mu$ such that for every $i<\mu, H_{i}:=\{\alpha \in \Gamma \mid h(\alpha)=i\}$ is stationary. By a club-guessing theorem due to Shelah [She94, §2] (see also [SS10]), we may fix a $C$-sequence $\vec{C}=\left\langle C_{\alpha}\right|$ $\alpha<\kappa\rangle$ such that:

○ For every $\alpha<\kappa, \operatorname{otp}\left(C_{\alpha}\right)=\operatorname{cf}(\alpha)$.

○ For every club $D \subseteq \kappa$ and every $i<\mu$, there exists $\gamma \in H_{i}$ with $\sup \left(\operatorname{nacc}\left(C_{\gamma}\right) \cap D\right)=\gamma$.

Note that $\operatorname{acc}\left(C_{\alpha}\right) \cap \Gamma=\emptyset$ for all $\alpha<\kappa$. Recalling Subsection 2.1, we now let $\operatorname{Tr}, \operatorname{tr}, \lambda$ and $\rho_{2}$ be the characteristic functions of walking along $\vec{C}$. In addition, we consider yet another function $\operatorname{tr}_{h}:[\kappa]^{2} \rightarrow{ }^{<\omega} \mu$ that is defined via $\operatorname{tr}_{h}(\alpha, \beta):=h \circ \operatorname{tr}(\alpha, \beta)$.

Fix a sequence $\left\langle Z_{\epsilon} \mid \epsilon<\kappa\right\rangle$ of elements of $[\mu]^{\mu}$ such that for every $(\alpha, \beta) \in[\mu]^{\mu},\left|Z_{\alpha} \cap Z_{\beta}\right|<\mu$.

Definition 4.6. For every ordinal $\xi<\mu$ and a pair $(\alpha, \beta) \in[\kappa]^{2}$, let

$$
\xi^{\alpha, \beta}:=\min \left\{n<\omega \mid \xi \in Z_{\operatorname{Tr}(\alpha, \beta)(n)} \text { or } n=\rho_{2}(\alpha, \beta)+1\right\} .
$$

Lemma 4.7. There exists a map $d_{1}:{ }^{<\omega} \mu \rightarrow \omega \times \mu \times \mu \times \mu$, such that for every $(\tau, \xi, \phi) \in \mu \times \mu \times \mu$ and every sequence $\left\langle\left(u_{i}, v_{i}, \sigma_{i}\right) \mid i<\mu\right\rangle$, with

1. $u_{i}$ and $v_{i}$ are nonempty elements of $\left[{ }^{<\omega} \mu\right]^{<\chi}$;

2. $i \in \operatorname{Im}(\varrho)$ for all $\varrho \in u_{i}$;

3. $\sigma_{j}{ }^{-}\langle j\rangle \sqsubseteq \sigma$ for all $\sigma \in v_{j}$,

there exist $(i, j) \in[\mu]^{2}$ satisfying that $d_{1}\left(\varrho^{-} \sigma\right)=(\ell(\varrho), \tau, \xi, \phi)$ for all $\varrho \in u_{i}$ and $\sigma \in v_{j}$.

Proof. Let $d:{ }^{<\omega} \mu \rightarrow \omega \times \mu \times \mu \times \mu$ be given by Fact 3.2 using $v:=\chi$. Fix a bijection $\pi: \mu \leftrightarrow \mu \times \mu \times \mu$. Then, define $d_{1}:{ }^{<\omega} \mu \rightarrow \omega \times \mu \times \mu \times \mu$ by letting $d_{1}(\sigma):=(n, \tau, \xi, \phi)$ whenever $d(\sigma)=(n, i, j, \gamma)$ and $\pi(\gamma)=(\tau, \xi, \phi)$. Evidently, $d_{1}$ is as sought.

Let $d_{1}:{ }^{<\omega} \mu \rightarrow \omega \times \mu \times \mu \times \mu$ be given by the preceding lemma. For every nonzero $\epsilon<\kappa$, fix a surjection $\psi_{\epsilon}: \mu \rightarrow \epsilon$. We are now ready to define our transformation.

Definition 4.8. Define $\mathbf{t}:[\kappa]^{2} \rightarrow[\kappa]^{3}$ by letting, for all $(\alpha, \beta) \in[\kappa]^{2}, \mathbf{t}(\alpha, \beta):=\left(\tau^{*}, \alpha^{*}, \beta^{*}\right)$ provided that, for $(n, \tau, \xi, \phi):=d_{1}\left(\operatorname{tr}_{h}(\alpha, \beta)\right)$, all of the following conditions are met:

$\circ \beta^{*}=\operatorname{Tr}(\alpha, \beta)(n)$ is $>\alpha$,

○ $\eta:=\psi_{\beta^{*}}(\phi)$ satisfies that $\eta+1<\alpha$,

$\circ \alpha^{*}=\operatorname{Tr}(\eta+1, \alpha)\left(\xi^{\eta+1, \alpha}\right)$, and

$\circ \tau^{*}=\tau<\alpha^{*}$. 
Otherwise, let $\mathbf{t}(\alpha, \beta):=(0, \alpha, \beta)$.

To verify that $\mathbf{t}$ witnesses $\mathrm{P} \ell_{1}(\kappa, \mu, \chi)$, suppose that we are given a family $\mathcal{A} \subseteq[\kappa]^{<\chi}$ consisting of $\kappa$ many pairwise disjoint sets.

Lemma 4.9. For every $i<\mu$, there exist an ordinal $\zeta_{i}<\kappa$ and a sequence $\overrightarrow{x^{i}}=\left\langle x_{\gamma}^{i} \mid \gamma \in \Gamma_{i}\right\rangle$ such that:

- $\Gamma_{i}$ is a stationary subset of $\kappa$.

○ for all $\gamma \in \Gamma_{i}, x_{\gamma}^{i} \in \mathcal{A}$ with $\min \left(x_{\gamma}^{i}\right)>\gamma$.

○ for all $\gamma \in \Gamma_{i}$ and $\beta \in x_{\gamma}^{i}, \lambda(\gamma, \beta)=\zeta_{i}$ and $i \in \operatorname{Im}\left(\operatorname{tr}_{h}(\gamma, \beta)\right)$.

Proof. Let $i<\mu$. By the pressing down lemma, it suffices to prove that, for every club $D \subseteq \kappa$, there exist $\gamma \in D, \zeta<\gamma$ and $x \in \mathcal{A}$ with $\min (x)>\gamma$ such that $\lambda(\gamma, \beta)=\zeta$ and $i \in \operatorname{tr}_{h}(\gamma, \beta)$ for all $\beta \in x$. Thus, let $D$ be an arbitrary club in $\kappa$.

By the choice of $\vec{C}$, fix $\delta \in H_{i}$ such that $\sup \left(\operatorname{nacc}\left(C_{\delta}\right) \cap D\right)=\delta$. Then, fix any $x \in \mathcal{A}$ with $\min (x)>\delta$. As $\delta \in \Gamma$ and $|x|<\chi<\operatorname{cf}(\delta)$, Fact 2.6 entails that we may find a large enough $\gamma \in \operatorname{nacc}\left(C_{\delta}\right) \cap D$ with $\zeta:=\sup \left(C_{\delta} \cap \gamma\right)$ being greater than $\sup _{\beta \in x} \lambda(\delta, \beta)$. Now, for every $\beta \in x$, we have $\lambda(\delta, \beta)<\zeta<\gamma<\delta<\beta$, so, by Fact 2.7, $\operatorname{tr}(\gamma, \beta)=\operatorname{tr}(\delta, \beta)^{-} \operatorname{tr}(\gamma, \delta)$. In particular, $i=h(\delta) \in \operatorname{Im}\left(\operatorname{tr}_{h}(\gamma, \beta)\right)$ Next, by Lemma 2.9, $\lambda(\gamma, \beta)=\max \{\lambda(\delta, \beta), \lambda(\gamma, \delta)\}$. As $\gamma \in C_{\delta}$, we have $\lambda(\gamma, \delta)=\sup \left(C_{\delta} \cap \gamma\right)>\zeta=\lambda(\delta, \beta)$, so that, altogether, $\lambda(\gamma, \beta)=\zeta$.

For each $i<\mu$, let $\zeta_{i}$ and $\vec{x}^{i}=\left\langle x_{\gamma}^{i} \mid \gamma \in \Gamma_{i}\right\rangle$ be given by the preceding lemma. For notational simplicity, we shall drop the superscript $i$, writing $\vec{x}^{i}=\left\langle x_{\gamma} \mid \gamma \in \Gamma_{i}\right\rangle .{ }^{3}$ Set $\zeta:=\sup _{i<\mu} \zeta_{i}$.

Definition 4.10. For $\eta<\kappa$ and $\xi, \phi<\mu, S_{\eta, \xi, \phi}$ denotes the set of all $\epsilon \in \Gamma$ with the property that, for every $\varsigma<\kappa$, there exists a sequence $\left\langle\beta_{i} \mid i<\mu\right\rangle \in \prod_{i<\mu} \Gamma_{i} \backslash \varsigma$ such that for all $i<\mu$ and $\beta \in x_{\beta_{i}}$ :

(i) $\operatorname{tr}(\epsilon, \beta)=\operatorname{tr}\left(\beta_{i}, \beta\right)^{-} \operatorname{tr}\left(\epsilon, \beta_{i}\right)$.

(ii) $\lambda(\epsilon, \beta)<\epsilon$.

(iii) If $i=0$, then $\lambda(\epsilon, \beta)=\eta=\psi_{\epsilon}(\phi)$, and $\rho_{2}(\epsilon, \beta)=\xi^{\epsilon, \beta}$.

Lemma 4.11. There exist $\eta<\kappa$ and $\xi, \phi<\mu$ for which $S_{\eta, \xi, \phi}$ is stationary.

Proof. For all $i<\mu$ and $\varsigma<\kappa$, denote $\beta_{i}^{\varsigma}:=\min \left(\Gamma_{i} \backslash \varsigma\right)$.

Let $\epsilon \in \Gamma \backslash(\zeta+1)$. For every $S$ in the interval $(\epsilon, \kappa)$, define $f_{\epsilon}^{S}: \mu \rightarrow \epsilon$ via $f_{\epsilon}^{S}(i):=$ $\max \left\{\zeta_{i}, \lambda\left(\epsilon, \beta_{i}^{\varsigma}\right)\right\}$. Now, find $\eta_{\epsilon}<\epsilon$ and $\phi_{\epsilon}, \xi_{\epsilon}<\mu$ for which

$$
\Sigma_{\epsilon}:=\left\{\varsigma \in(\epsilon, \kappa) \mid f_{\epsilon}^{\varsigma}(0)=\eta_{\epsilon}=\psi_{\epsilon}\left(\phi_{\epsilon}\right) \& \xi_{\epsilon} \in Z_{\epsilon} \backslash \bigcup\left\{Z_{\tau} \mid \tau \in \operatorname{Im}(\operatorname{tr}(\epsilon, \beta)), \beta \in x_{\beta_{0}^{\varsigma}}\right\}\right\}
$$

is cofinal in $\kappa$.

Finally, find $\eta, \xi, \phi$ for which $S:=\left\{\epsilon \in \Gamma \backslash(\zeta+1) \mid(\eta, \xi, \phi)=\left(\eta_{\epsilon}, \xi_{\epsilon}, \phi_{\epsilon}\right)\right\}$ is stationary. We claim that $S \subseteq S_{\eta, \xi, \phi}$. Let $\epsilon \in S$ be arbitrary; to see that $\epsilon \in S_{\eta, \xi, \phi}$, let $\varsigma<\kappa$ be arbitrary. By increasing $\varsigma$, we may assume that $\varsigma \in \Sigma_{\epsilon}$. Let $i<\mu$ and $\beta \in x_{\beta_{i}^{\varsigma}}$ be arbitrary. We will show that:

(i') $\operatorname{tr}(\epsilon, \beta)=\operatorname{tr}\left(\beta_{i}^{S}, \beta\right)^{-} \operatorname{tr}\left(\epsilon, \beta_{i}^{S}\right)$.

(ii') $\lambda(\epsilon, \beta)=f_{\epsilon}^{S}(i)$.

(iii') if $i=0$, then $\rho_{2}(\epsilon, \beta)=\xi^{\epsilon, \beta}$.

As $\lambda\left(\beta_{i}^{S}, \beta\right)=\zeta_{i}<\epsilon<\beta_{i}^{S}<\beta$, it follows from Fact 2.7 that Clause (i') is satisfied, and it follows from Lemma 2.9 that

$$
\lambda(\epsilon, \beta)=\max \left\{\lambda\left(\beta_{i}^{S}, \beta\right), \lambda\left(\epsilon, \beta_{i}^{S}\right)\right\}=\max \left\{\zeta_{i}, \lambda\left(\epsilon, \beta_{i}^{S}\right)\right\}=f_{\epsilon}^{S}(i) .
$$

In addition, from $\varsigma \in \Sigma_{\epsilon}$, Clause (iii') is satisfied.

${ }^{3}$ This is formally legitimate provided that the stationary sets in $\left\langle\Gamma_{i} \mid i<\mu\right\rangle$ are pairwise disjoint. Now, as $\mu$ is regular, for any sequence $\left\langle\Gamma_{i} \mid i<\mu\right\rangle$ of stationary subsets of $\mu^{+}$, there exists a sequence $\left\langle\bar{\Gamma}_{i} \mid i<\mu\right\rangle$ of pairwise disjoint stationary sets such that $\bar{\Gamma}_{i} \subseteq \Gamma_{i}$ for all $i<\mu$ (see [IR21]). So, we may as well assume that the original sequence consists of pairwise disjoint sets. 
Let $\eta, \xi, \phi$ be given by the preceding lemma. Let $D$ be a club in $\kappa$ such that for all $\delta \in D$, there exists $M_{\delta} \prec \mathcal{H}_{\kappa^{+}}$containing the parameter $p:=\left\{\Gamma, S_{\eta, \xi, \phi}, \overrightarrow{x^{0}}, \vec{C}, h, \mu\right\}$ and satisfying $M_{\delta} \cap \kappa=\delta$. Finally, let

$$
S^{*}:=S_{\eta, \xi, \phi} \cap \bigcap_{j<\mu} \operatorname{acc}^{+}\left(H_{j} \cap D\right) .
$$

Lemma 4.12. Let $\left(\tau^{*}, \alpha^{*}, \beta^{*}\right) \in \mu \circledast S^{*} \circledast S^{*}$. There exists $(a, b) \in[\mathcal{A}]^{2}$ such that $\mathbf{t}[a \times b]=$ $\left\{\left(\tau^{*}, \alpha^{*}, \beta^{*}\right)\right\}$.

Proof. As $\beta^{*} \in S^{*} \subseteq S_{\eta, \xi, \phi}$, let us fix a sequence $\left\langle\beta_{i} \mid i<\mu\right\rangle \in \prod_{i<\mu} \Gamma_{i} \backslash\left(\beta^{*}+1\right)$ such that for all $i<\mu$ and $\beta \in x_{\beta_{i}}$ :

1. $\operatorname{tr}\left(\beta^{*}, \beta\right)=\operatorname{tr}\left(\beta_{i}, \beta\right)^{-} \operatorname{tr}\left(\beta^{*}, \beta_{i}\right)$.

2. $\lambda\left(\beta^{*}, \beta\right)<\beta^{*}$.

3. $\psi_{\beta^{*}}(\phi)=\eta$.

For each $i<\mu,\left|x_{\beta_{i}}\right|<\chi<\operatorname{cf}\left(\beta^{*}\right)$, so we may define a function $f: \mu \rightarrow \beta^{*}$ via $f(i):=$ $\sup \left\{\lambda\left(\beta^{*}, \beta\right) \mid \beta \in x_{\beta_{i}}\right\}$. For all $j<\mu$, as $\beta^{*} \in \Gamma \cap \operatorname{acc}^{+}\left(H_{j} \cap D\right)$, we may pick a large enough $\delta_{j} \in H_{j} \cap D \cap \beta^{*}$ such that $\delta_{j}>\max \left\{\alpha^{*}, \sup _{i<j} f(i)\right\}$. As $M_{\delta_{j}}$ contains $p$, we have that $S_{\eta, \xi, \phi} \in M_{\delta_{j}}$. As $\delta_{j} \in \Gamma$, Fact 2.6 entails that $\varsigma_{j}:=\max \left\{\alpha^{*}, \sup _{i<j} f(i), \lambda\left(\delta_{j}, \beta^{*}\right)\right\}+1$ is smaller than $\delta_{j}$. Since $\alpha^{*} \in M_{\delta_{j}} \cap S_{\eta, \xi, \phi}$, we may then find $\alpha_{j} \in M_{\delta_{j}} \cap \Gamma_{0} \backslash \varsigma_{j}$ such that for all $\alpha \in x_{\alpha_{j}}$ :

(4) $\lambda\left(\alpha^{*}, \alpha\right)=\eta$ and $\rho_{2}\left(\alpha^{*}, \alpha\right)=\xi^{\alpha^{*}, \alpha}$.

Note that from $\alpha_{j} \in M_{\delta_{j}}$, it follows that $\sup \left(x_{\alpha_{j}}\right)<\delta_{j}$. Write $a_{j}:=x_{\alpha_{j}}$ and $b_{i}:=x_{\beta_{i}}$. Fix arbitrary $(i, j) \in[\mu]^{2}$ and $(\alpha, \beta) \in a_{j} \times b_{i}$. Then:

$$
\eta+1<\alpha^{*} \leq \max \left\{\alpha^{*}, \lambda\left(\beta^{*}, \beta\right), \lambda\left(\delta_{j}, \beta^{*}\right)\right\} \leq \varsigma_{j} \leq \alpha_{j}<\alpha<\delta_{j}<\beta^{*}<\beta_{i}<\beta .
$$

In particular, Fact 2.7 yields the following conclusions:

(a) From $\lambda\left(\beta^{*}, \beta\right)<\alpha<\beta^{*}<\beta$, we have $\operatorname{tr}(\alpha, \beta)=\operatorname{tr}\left(\beta^{*}, \beta\right)^{-} \operatorname{tr}\left(\alpha, \beta^{*}\right)$.

(b) From $\lambda\left(\delta_{j}, \beta^{*}\right)<\alpha<\delta_{j}<\beta^{*}$, we have $\operatorname{tr}\left(\alpha, \beta^{*}\right)=\operatorname{tr}\left(\delta_{j}, \beta^{*}\right)^{-} \operatorname{tr}\left(\alpha, \delta_{j}\right)$.

Altogether,

$$
\operatorname{tr}(\alpha, \beta)=\operatorname{tr}\left(\beta_{i}, \beta\right)^{-} \operatorname{tr}\left(\beta^{*}, \beta_{i}\right)^{-} \operatorname{tr}\left(\delta_{j}, \beta^{*}\right)^{-} \operatorname{tr}\left(\alpha, \delta_{j}\right) .
$$

For each $i<\mu$, set $u_{i}:=\left\{\operatorname{tr}_{h}\left(\beta^{*}, \beta\right) \mid \beta \in b_{i}\right\}$. As $\beta_{i} \in \Gamma_{i}$, Clause (1) above implies that $i \in \operatorname{Im}(\varrho)$ for all $\varrho \in u_{i}$. For each $j<\mu$, set $v_{j}:=\left\{\operatorname{tr}_{h}\left(\alpha, \beta^{*}\right) \mid \alpha \in a_{j}\right\}$ and $\sigma_{j}:=\operatorname{tr}_{h}\left(\delta_{j}, \beta^{*}\right)$. As $\delta_{j} \in H_{j}$, we infer that $\sigma_{j}{ }^{\curlyvee}\langle j\rangle \sqsubseteq \sigma$ for all $\sigma \in v_{j}$.

Next, by the choice of $d_{1}$, fix $(i, j) \in[\mu]^{2}$ such that $d_{1}\left(\varrho^{-} \sigma\right)=\left(\ell(\varrho), \tau^{*}, \xi, \phi\right)$ for all $\varrho \in u_{i}$ and $\sigma \in v_{j}$. Set $a:=a_{j}$ and $b:=b_{i}$, so that $(a, b) \in[\mathcal{A}]^{2}$.

To see that $\mathbf{t}[a \times b]=\left\{\left(\tau^{*}, \alpha^{*}, \beta^{*}\right)\right\}$, fix arbitrary $\alpha \in a$ and $\beta \in b$. Denote $\varrho:=\operatorname{tr}_{h}\left(\beta^{*}, \beta\right)$ and $\sigma:=\operatorname{tr}_{h}\left(\alpha, \beta^{*}\right)$, so that $\varrho \in u_{i}$ and $\sigma \in v_{j}$. Then $d_{1}\left(\operatorname{tr}_{h}(\alpha, \beta)\right)=\left(\ell(\varrho), \tau^{*}, \xi, \phi\right)$, so that

○ $\operatorname{Tr}(\alpha, \beta)(\ell(\varrho))=\operatorname{Tr}(\alpha, \beta)\left(\rho_{2}\left(\beta^{*}, \beta\right)\right)=\beta^{*}$.

○ $\eta=\psi_{\beta^{*}}(\phi)$ and $\eta+1<\alpha$.

$\circ \tau^{*}<\alpha^{*}$.

Now, since $\lambda\left(\alpha^{*}, \alpha\right)=\eta<\eta+1<\alpha^{*}<\alpha, \operatorname{tr}(\eta+1, \alpha)=\operatorname{tr}\left(\alpha^{*}, \alpha\right)^{-} \operatorname{tr}\left(\eta+1, \alpha^{*}\right)$. So, since $\rho_{2}\left(\alpha^{*}, \alpha\right)=\xi^{\alpha^{*}, \alpha}, \rho_{2}\left(\alpha^{*}, \alpha\right)=\xi^{\eta+1, \alpha}$ and $\alpha^{*}=\operatorname{Tr}(\eta+1, \alpha)\left(\xi^{\eta+1, \alpha}\right)$.

\section{Clause (1) of Theorem $C$}

In this section, we suppose that $\square(\kappa)$ holds. Fix an arbitrary $\chi \in \operatorname{Reg}(\kappa)$ with $\chi^{+}<\kappa$. We shall construct a witness to $\mathrm{P} \ell_{1}(\kappa, \kappa, \chi)$. Denote $\mu:=\chi^{+}$. 
Lemma 5.1. There exists a $C$-sequence $\vec{C}=\left\langle C_{\alpha} \mid \alpha<\kappa\right\rangle$ satisfying the following:

1. $C_{\alpha+1}=\{0, \alpha\}$ for every $\alpha<\kappa$.

2. for every club $D \subseteq \kappa$, there exists $\delta \in E_{\neq \mu}^{\kappa}$ with $\sup \left(\operatorname{nacc}\left(C_{\delta}\right) \cap D\right)=\delta$.

3. for every $\alpha \in \operatorname{acc}(\kappa)$ and $\bar{\alpha} \in \operatorname{acc}\left(C_{\alpha}\right), C_{\bar{\alpha}}=C_{\alpha} \cap \bar{\alpha}$.

4. for every $\gamma<\kappa,\left\{\delta \in E_{\chi}^{\kappa} \mid \min \left(C_{\delta}\right)=\gamma\right\}$ is stationary.

Proof. As $\square(\kappa)$ holds, we may appeal to [Rin17, Proposition 3.5] with $S:=E_{\neq \mu}^{\kappa}$, and obtain a $C$ sequence $\vec{C}$ satisfying Clauses (2) and (3). In particular, $\vec{C}$ is a $\square(\kappa)$-sequence. Now, by feeding $\Gamma:=E_{\chi}^{\kappa}$ and $\vec{C}$ to the proof of [Rin14a, Proposition 3.2], we obtain a $C$-sequence $\left\langle\bar{C}_{\alpha} \mid \alpha<\kappa\right\rangle$ satisfying Clauses (1), (3) and (4). An inspection of the said proof makes clear that $\sup \left(\bar{C}_{\alpha} \Delta C_{\alpha}\right)<\alpha$ for every $\alpha \in \operatorname{acc}(\kappa)$, so that Clause (2) is valid for $\left\langle\bar{C}_{\alpha} \mid \alpha<\kappa\right\rangle$, as well.

Let $\vec{C}$ be given by the preceding lemma. Recalling Subsection 2.1, we now let $\operatorname{Tr}, \operatorname{tr}, \lambda$ and $\rho_{2}$ be the characteristic functions of walking along $\vec{C}$, and let $\eta_{\alpha, \beta}$ be the notation established in Definition 2.12.

Fix a bijection $\pi: \kappa \leftrightarrow \kappa \times \kappa$. Define a function $g: \kappa \rightarrow \kappa \times \kappa$ via $g(\alpha):=\pi\left(\min \left(C_{\alpha}\right)\right)$. Define a function $h: \kappa \rightarrow \mu$ by letting $h(\alpha):=\min \left(C_{\alpha}\right)$ for all $\alpha<\kappa$ with $\min \left(C_{\alpha}\right)<\mu$, and $h(\alpha):=0$, otherwise. Then, define a function $\operatorname{tr}_{h}:[\kappa]^{2} \rightarrow{ }^{<\omega} \mu$ via $\operatorname{tr}_{h}(\alpha, \beta):=h \circ \operatorname{tr}(\alpha, \beta)$. Also, for each $(\eta, \tau) \in \kappa \times \kappa$, denote $G_{\eta, \tau}:=\{\delta<\kappa \mid g(\delta)=(\eta, \tau)\}$, and for each $i<\mu$, denote $H_{i}:=h^{-1}\{i\}$.

Lemma 5.2. For every $(\delta, \beta) \in[\kappa]^{2}, C_{\delta}=C_{\check{\circlearrowright}_{\delta, \beta}} \cap \delta$. In particular:

$\circ h(\delta)=h\left(\delta_{\delta, \beta}\right)$.

$\circ$ for every $\epsilon<\delta, \lambda(\epsilon, \delta)=\lambda\left(\epsilon, \partial_{\delta, \beta}\right)$.

Proof. By Lemma 5.1(3) together with Lemma 2.11(2).

Exactly as in Subsection 4.1, we appeal to Lemma 3.3 to fix a map $d_{0}:<\omega \mu \rightarrow \omega$, its corresponding $\mu$-additive proper ideal $J$, define a coloring $c:[\kappa]^{2} \rightarrow \kappa \times \kappa$ via

$$
c(\alpha, \beta):=g\left(\operatorname{Tr}(\alpha, \beta)\left(d_{0}\left(\operatorname{tr}_{h}(\alpha, \beta)\right)\right)\right),
$$

and define the sets $S_{\eta}$ and the transformation $\mathbf{t}$ in the very same way.

Definition 5.3. For $\eta<\kappa, S_{\eta}$ denotes the set of all $\epsilon<\kappa$ with the property that, for every $\varsigma<\kappa$, there exist $I \in J^{+}$and a sequence $\left\langle\beta_{i} \mid i \in I\right\rangle \in \prod_{i \in I} H_{i} \backslash \varsigma$, such that for all $i \in I$ and $\beta \in x_{\beta_{i}}$ :

(i) $i \in \operatorname{Im}\left(\operatorname{tr}_{h}(\epsilon, \beta)\right)$.

(ii) $\lambda(\epsilon, \beta)=\eta$.

(iii) $\rho_{2}(\epsilon, \beta)=\eta_{\epsilon, \beta}$.

Definition 5.4. Define $\mathbf{t}:[\kappa]^{2} \rightarrow[\kappa]^{3}$ by letting, for all $(\alpha, \beta) \in[\kappa]^{2}, \mathbf{t}(\alpha, \beta):=\left(\tau, \alpha^{*}, \beta^{*}\right)$ provided that the following conditions are met:

$\circ(\eta, \tau):=c(\alpha, \beta)$ and $\max \{\eta+1, \tau\}<\alpha$,

○ $\beta^{*}=\operatorname{Tr}(\alpha, \beta)\left(\eta_{\alpha, \beta}\right)$ is $>\alpha$, and

$\circ \alpha^{*}=\operatorname{Tr}(\eta+1, \alpha)\left(\eta_{\eta+1, \alpha}\right)$.

Otherwise, let $\mathbf{t}(\alpha, \beta):=(0, \alpha, \beta)$.

To verify that $\mathbf{t}$ witnesses $\mathrm{P} \ell_{1}(\kappa, \kappa, \chi)$, suppose that we are given a family $\mathcal{A} \subseteq[\kappa]^{<\chi}$ consisting of $\kappa$ many pairwise disjoint sets. Fix a sequence $\vec{x}=\left\langle x_{\delta} \mid \delta<\kappa\right\rangle$ such that for all $\delta<\kappa, x_{\delta} \in \mathcal{A}$ with $\min \left(x_{\delta}\right)>\delta$.

Lemma 5.5. There exists $\eta<\kappa$ for which $S_{\eta}$ is stationary.

Proof. It suffices to prove that, for every club $D \subseteq \kappa$, there exist $\epsilon \in D$ and $\eta<\epsilon$ for which $\epsilon \in S_{\eta}$.

Thus, let $D$ be an arbitrary club in $\kappa$. 
Define a function $f: E_{\chi}^{\kappa} \rightarrow \kappa$ via

$$
f(\delta):=\sup \left\{\lambda\left(\partial_{\delta, \beta}, \beta\right) \mid \beta \in x_{\delta}\right\} .
$$

As $\left|x_{\delta}\right|<\chi=\operatorname{cf}(\delta)$, Lemma 2.11(1) entails that $f$ is regressive. So, for all $i<\mu$, let us pick a stationary subset $\bar{H}_{i} \subseteq H_{i}$ such that $f \uparrow \bar{H}_{i}$ is constant. Set $\zeta:=\sup \left(f\left[\bigcup_{i<\mu} \bar{H}_{i}\right]\right)$. Now, by Lemma 5.1(2), let us pick a nonzero $\gamma \in E_{\neq \mu}^{\kappa}$ with $\sup \left(\operatorname{nacc}\left(C_{\gamma}\right) \cap(D \backslash \zeta)\right)=\gamma$.

Let $\varsigma<\kappa$. Fix a sequence $\left\langle\beta_{i}^{\varsigma} \mid i<\mu\right\rangle \in \prod_{i<\mu} \bar{H}_{i} \backslash \max \{\gamma+1, \varsigma\}$. For every $i<\mu$, let

$$
\zeta_{i}^{\varsigma}:= \begin{cases}0, & \text { if } \gamma \in \operatorname{acc}\left(C_{\beta_{i}^{\varsigma}}\right) \\ \sup \left(C_{\beta_{i}^{\varsigma}} \cap \gamma\right), & \text { if } \gamma \in \operatorname{nacc}\left(C_{\beta_{i}^{\varsigma}}\right) \\ \lambda\left(\partial_{\gamma, \beta_{i}^{\varsigma}}, \beta_{i}^{\varsigma}\right), & \text { otherwise. }\end{cases}
$$

Note that, by Lemma 2.11(1), $\zeta_{i}^{\varsigma}<\gamma$.

As $\operatorname{cf}(\gamma) \neq \mu$ and as $J$ is a $\mu$-additive proper ideal on $\mu$, we may now fix $I^{\varsigma} \in J^{+}$along with some ordinal $\xi^{\varsigma}<\gamma$ such that $\max \left\{\zeta, \zeta_{i}^{\varsigma}\right\} \leq \xi^{\varsigma}$ for all $i \in I^{\varsigma}$. Then, pick a large enough $\epsilon^{\varsigma} \in \operatorname{nacc}\left(C_{\gamma}\right) \cap D$ such that $\sup \left(C_{\gamma} \cap \epsilon^{\varsigma}\right)>\xi^{\varsigma}$.

Fix $\epsilon \in \operatorname{nacc}\left(C_{\gamma}\right) \cap D$ for which $\Sigma:=\left\{\varsigma<\kappa \mid \epsilon^{\varsigma}=\epsilon\right\}$ is cofinal in $\kappa$. Denote $\eta:=\sup \left(C_{\gamma} \cap \epsilon\right)$, so that $\eta<\epsilon$. We have $\epsilon \in D$. To see that $\epsilon \in S_{\eta}$, let $\varsigma<\kappa$ be arbitrary. By increasing $\varsigma$, we may assume that $\varsigma \in \Sigma$. Let $i \in I^{\varsigma}$ and $\beta \in x_{\beta_{i}^{\varsigma}}$ be arbitrary. We must show that:

(i) $i \in \operatorname{Im}\left(\operatorname{tr}_{h}(\epsilon, \beta)\right)$.

(ii) $\lambda(\epsilon, \beta)=\eta$.

(iii) $\rho_{2}(\epsilon, \beta)=\eta_{\epsilon, \beta}$.

We have:

$$
\lambda\left(\partial_{\beta_{i}^{\varsigma}, \beta}, \beta\right) \leq f\left(\beta_{i}^{\varsigma}\right) \leq \zeta \leq \xi^{\varsigma}<\eta<\epsilon<\gamma<\beta_{i}^{\varsigma}<\beta .
$$

It thus follows from Fact 2.7 that $\operatorname{tr}(\epsilon, \beta)=\operatorname{tr}\left(\mathrm{\partial}_{\beta_{i}^{\varsigma}, \beta}, \beta\right)^{-} \operatorname{tr}\left(\epsilon, \mathrm{\partial}_{\beta_{i}^{\varsigma}, \beta}\right)$. So, since $\beta_{i}^{\varsigma} \in H_{i}$, Lemma 5.2 implies that $i \in \operatorname{Im}\left(\operatorname{tr}_{h}(\epsilon, \beta)\right)$.

Claim 5.5.1. $\lambda(\epsilon, \beta)=\eta$ and $\rho_{2}(\epsilon, \beta)=\eta_{\epsilon, \beta}$.

Proof. By Lemma 2.9, $\lambda(\epsilon, \beta)=\max \left\{\lambda\left(\mathrm{\partial}_{\beta_{i}^{\varsigma}, \beta}, \beta\right), \lambda\left(\epsilon, \mathrm{\partial}_{\beta_{i}^{\varsigma}, \beta}\right)\right\}$. Now, there are three cases to consider:

- If $\gamma \in \operatorname{acc}\left(C_{\beta_{i}^{\varsigma}}\right)$, then $C_{\beta_{i}^{\varsigma}} \cap \gamma=C_{\gamma}$, and since $\epsilon \in C_{\gamma}, \operatorname{tr}(\epsilon, \beta)=\operatorname{tr}\left(\varlimsup_{\beta_{i}^{\varsigma}, \beta}, \beta\right)^{-}\left\langle\mathrm{\partial}_{\beta_{i}^{\varsigma}, \beta}\right\rangle$, and $\lambda\left(\epsilon, \mathrm{\partial}_{\beta_{i}^{\varsigma}, \beta}\right)=\sup \left(C_{\gamma} \cap \epsilon\right)=\eta>\zeta \geq \lambda\left(\mathrm{\partial}_{\beta_{i}^{\varsigma}, \beta}, \beta\right)$, so the conclusion follows.

- If $\gamma \in \operatorname{nacc}\left(C_{\beta_{i}^{\varsigma}}\right)$, then since $\epsilon \in C_{\gamma}, \operatorname{tr}(\epsilon, \beta)=\operatorname{tr}\left(\partial_{\beta_{i}^{\varsigma}, \beta}, \beta\right)^{-}\left\langle\mathrm{\partial}_{\beta_{i}^{\varsigma}, \beta}, \gamma\right\rangle$, so that $\lambda(\epsilon, \beta)=$ $\max \left\{\lambda\left(\mathrm{\partial}_{\beta_{i}^{\varsigma}, \beta}, \beta\right), \sup \left(C_{\mathrm{\partial}_{\beta_{i}^{S}, \beta}} \cap \epsilon\right), \sup \left(C_{\gamma} \cap \epsilon\right)\right\}=\max \left\{\lambda\left(\mathrm{\partial}_{\beta_{i}^{\varsigma}, \beta}, \beta\right), \zeta_{i}^{\varsigma}, \eta\right\}$, and the conclusion follows.

- Otherwise, $\partial_{\gamma, \beta_{i}^{\varsigma}} \neq \beta_{i}^{S}$. Then $\lambda\left(\partial_{\gamma, \beta_{i}^{\varsigma}}, \beta_{i}^{S}\right)=\zeta_{i}^{\varsigma} \leq \xi^{\varsigma}<\epsilon<\gamma \leq \partial_{\gamma, \beta_{i}^{\varsigma}}<\beta_{i}^{S}$, and so, by Fact $2.7, \operatorname{tr}\left(\epsilon, \beta_{i}^{S}\right)=\operatorname{tr}\left(\partial_{\gamma, \beta_{i}^{\varsigma}}, \beta_{i}^{\varsigma}\right)^{-} \operatorname{tr}\left(\epsilon, \partial_{\gamma, \beta_{i}^{\varsigma}}\right)$. Thus, by Lemma 2.9,

$$
\lambda\left(\epsilon, \beta_{i}^{\varsigma}\right)=\max \left\{\lambda\left(\partial_{\gamma, \beta_{i}^{\varsigma}}, \beta_{i}^{\varsigma}\right), \lambda\left(\epsilon, \partial_{\gamma, \beta_{i}^{\varsigma}}\right)\right\}=\max \left\{\zeta_{i}^{\varsigma}, \lambda\left(\epsilon, \partial_{\gamma, \beta_{i}^{\varsigma}}\right)\right\}
$$

By Lemma 5.2, $\lambda\left(\epsilon, \partial_{\beta_{i}^{\varsigma}, \beta}\right)=\lambda\left(\epsilon, \beta_{i}^{\varsigma}\right)$. As $\epsilon \in C_{\gamma}=C_{\delta_{\gamma, \beta_{i}^{\varsigma}}} \cap \gamma$, we get that $\lambda\left(\epsilon, \partial_{\gamma, \beta_{i}^{\varsigma}}\right)=$ $\sup \left(C_{\gamma} \cap \epsilon\right)=\eta$. Altogether, $\lambda(\epsilon, \beta)=\max \left\{\lambda\left(\partial_{\beta_{i}^{\varsigma}, \beta}, \beta\right), \zeta_{i}^{\varsigma}, \eta\right\}$. But, $\eta>\xi^{\varsigma} \geq \max \left\{\zeta, \zeta_{i}^{\varsigma}\right\} \geq$

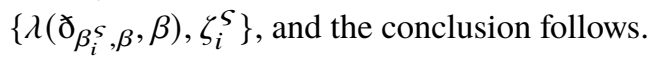

This completes the proof.

Let $\eta$ be given by the preceding lemma. Let $D$ be a club in $\kappa$ such that for all $\delta \in D$, there exists $M_{\delta} \prec \mathcal{H}_{\kappa^{+}}$containing the parameter $p:=\left\{S_{\eta}, \vec{x}, \vec{C}, h\right\}$ and satisfying $M_{\delta} \cap \kappa=\delta$. Consider the club 


$$
E:=\triangle_{\tau<\kappa} \operatorname{acc}^{+}\left(G_{\eta, \tau} \cap \bigcap_{j<\mu} \operatorname{acc}^{+}\left(H_{j} \cap D\right)\right) .
$$

Finally, let $S^{*}:=\left\{\epsilon \in S_{\eta} \mid \sup \left(E \cap \epsilon \backslash C_{\epsilon}\right)=\epsilon\right\}$.

Lemma 5.6. $S^{*}$ is stationary.

Proof. As $\vec{C}$ is a $\square(\kappa)$-sequence, [BR19, Lemma 1.23] implies that $\vec{C}$ is amenable in the sense of [BR19, Definition 1.3], so that $\left\{\epsilon \in \kappa \mid \sup \left(E \cap \epsilon \backslash C_{\epsilon}\right)<\epsilon\right\}$ is nonstationary.

Lemma 5.7. Let $\left(\tau^{*}, \alpha^{*}, \beta^{*}\right) \in \kappa \circledast S^{*} \circledast S^{*}$. There exists $(a, b) \in[\mathcal{A}]^{2}$ such that $\mathbf{t}[a \times b]=\left\{\left(\tau^{*}, \alpha^{*}, \beta^{*}\right)\right\}$.

Proof. As $\beta^{*} \in S^{*} \subseteq S_{\eta}$, let us pick $I \in J^{+}$and a sequence $\left\langle\beta_{i} \mid i \in I\right\rangle \in \prod_{i \in I} H_{i} \backslash\left(\beta^{*}+1\right)$ such that for all $i \in I$ and $\beta \in x_{\beta_{i}}$ :

1. $i \in \operatorname{tr}_{h}\left(\beta^{*}, \beta\right)$.

2. $\lambda\left(\beta^{*}, \beta\right)=\eta$.

3. $\rho_{2}\left(\beta^{*}, \beta\right)=\eta_{\beta^{*}, \beta}$.

Denote $G:=G_{\eta, \tau^{*}} \cap \bigcap_{j<\mu} \operatorname{acc}^{+}\left(H_{j} \cap D\right)$. From $\beta^{*} \in S^{*}$ and as $C_{\beta^{*}}$ is closed, it follows that $\sup \left(G \cap \beta^{*} \backslash C_{\beta^{*}}\right)=\beta^{*}$. Thus, we pick a large enough $\gamma \in G \cap \beta^{*} \backslash C_{\beta^{*}}$ such that $\sup \left(C_{\beta^{*}} \cap \gamma\right)>\alpha^{*}$. In particular, for $\varepsilon:=\mathrm{\partial}_{\gamma, \beta^{*}}, \lambda\left(\varepsilon, \beta^{*}\right)>\alpha^{*}>\eta$.

For each $j<\mu$, as $\gamma \in G \subseteq \operatorname{acc}^{+}\left(H_{j} \cap D\right)$, Lemma 2.11(1) entails that we may pick a large enough $\delta_{j} \in H_{j} \cap D \cap \gamma$ such that $\delta_{j}>\lambda\left(\varepsilon, \beta^{*}\right)$. As $M_{\delta_{j}}$ contains $p$, we have that $S_{\eta} \in M_{\delta_{j}}$. By Lemma 2.11(1), $\varsigma_{j}:=\max \left\{\alpha^{*}, \lambda\left(\varepsilon, \beta^{*}\right), \lambda\left(\partial_{\delta_{j}, \gamma}, \gamma\right)\right\}+1$ is smaller than $\delta_{j} .{ }^{4}$ Since $\alpha^{*} \in M_{\delta_{j}} \cap S_{\eta}$, we may then find $\alpha_{j} \in M_{\delta_{j}} \cap\left(\bigcup_{i<\mu} H_{i}\right) \backslash \varsigma_{j}$ such that for all $\alpha \in x_{\alpha_{j}}$ :

(2') $\lambda\left(\alpha^{*}, \alpha\right)=\eta$.

(3') $\rho_{2}\left(\alpha^{*}, \alpha\right)=\eta_{\alpha^{*}, \alpha}$.

Note that from $\alpha_{j} \in M_{\delta_{j}}$, it follows that $\sup \left(x_{\alpha_{j}}\right)<\delta_{j}$. Write $a_{j}:=x_{\alpha_{j}}$ and $b_{i}:=x_{\beta_{i}}$. Let $(i, j, \alpha, \beta) \in I \times \mu \times a_{j} \times b_{i}$ be arbitrary. Then:

$$
\eta<\eta+1<\alpha^{*}<\varsigma_{j} \leq \alpha_{j}<\alpha<\delta_{j}<\gamma \leq \varepsilon<\beta^{*}<\beta_{i}<\beta .
$$

In particular, Fact 2.7 yields the following conclusions:

(a) From $\lambda\left(\beta^{*}, \beta\right)=\eta<\alpha<\beta^{*}<\beta$, we have $\operatorname{tr}(\alpha, \beta)=\operatorname{tr}\left(\beta^{*}, \beta\right)^{-} \operatorname{tr}\left(\alpha, \beta^{*}\right)$.

(b) From $\lambda\left(\varepsilon, \beta^{*}\right)<\varsigma_{j}<\alpha<\beta^{*}$, we have $\operatorname{tr}\left(\alpha, \beta^{*}\right)=\operatorname{tr}\left(\varepsilon, \beta^{*}\right)^{-} \operatorname{tr}(\alpha, \varepsilon)$.

(c) From $\lambda\left(\delta_{\delta_{j}, \gamma}, \varepsilon\right)=\lambda\left(\partial_{\delta_{j}, \gamma}, \gamma\right)<\varsigma_{j}<\alpha<\delta_{j} \leq \succsim_{\delta_{j}, \gamma} \leq \gamma \leq \varepsilon$, we have $\operatorname{tr}(\alpha, \varepsilon)=\operatorname{tr}\left(\partial_{\delta_{j}, \gamma}, \varepsilon\right)^{-} \operatorname{tr}\left(\alpha, \partial_{\delta_{j}, \gamma}\right)$.

So that, altogether,

$$
\operatorname{tr}(\alpha, \beta)=\operatorname{tr}\left(\beta^{*}, \beta\right)^{-} \operatorname{tr}\left(\varepsilon, \beta^{*}\right)^{-} \operatorname{tr}\left(\succsim_{\delta_{j}, \gamma}, \varepsilon\right)^{-} \operatorname{tr}\left(\alpha, \partial_{\delta_{j}, \gamma}\right) .
$$

In addition, from $\lambda\left(\alpha^{*}, \alpha\right)=\eta<\eta+1<\alpha^{*}<\alpha$, we infer that

(d) $\operatorname{tr}(\eta+1, \alpha)=\operatorname{tr}\left(\alpha^{*}, \alpha\right)^{-} \operatorname{tr}\left(\eta+1, \alpha^{*}\right)$.

For each $i \in I$, denote $u_{i}:=\left\{\operatorname{tr}_{h}(\varepsilon, \beta) \mid \beta \in b_{i}\right\}$. By Clause (1) above, for all $\varrho \in u_{i}, i \in$ $\operatorname{Im}\left(\operatorname{tr}_{h}\left(\beta^{*}, \beta\right)\right) \subseteq \operatorname{Im}(\varrho)$.

For each $j<\mu$, denote $v_{j}:=\left\{\operatorname{tr}_{h}(\alpha, \varepsilon) \mid \alpha \in a_{j}\right\}$. By Clause (c) above, for all $\sigma \in v_{j}$, $\operatorname{tr}_{h}\left(\partial_{\delta_{j}, \gamma}, \varepsilon\right)^{-}\langle j\rangle \sqsubseteq \sigma$.

Next, by the choice of $d_{0}$, fix $(i, j) \in[I]^{2}$ such that $d_{0}\left(\varrho^{-} \sigma\right)=\ell(\varrho)$ for all $\varrho \in u_{i}$ and $\sigma \in v_{j}$. Set $a:=x_{j}$ and $b:=x_{i}$. The rest of the proof is now identical to that of Lemma 4.5.

${ }^{4}$ By Convention 2.8, if $\partial_{\delta_{j}, \gamma}=\gamma$, then $\lambda\left({ }_{\delta_{j}, \gamma}, \gamma\right)=0$. 


\section{Clause (3) of Theorem $\mathrm{C}$}

In this section, we suppose that $\kappa$ is inaccessible, $\chi \in \operatorname{Reg}(\kappa)$, and $E_{\geq \chi}^{\kappa}$ admits a stationary set that does not reflect at inaccessibles. Let $\mu:=\chi^{+}$. We shall prove that $\mathrm{P} \ell_{1}(\kappa, \mu, \chi)$ holds. Note that by the result of Section 4, we may assume that every stationary subset of $E_{\geq \chi}^{\kappa}$ reflects.

Lemma 6.1. There exist $\sigma^{1}, \sigma^{0} \in \operatorname{Reg}(\kappa)$ with $\mu<\sigma^{1}<\sigma^{0}$ and stationary subsets $S^{1}, S^{0}$ of $\kappa$ consisting of singular cardinals such that

- $S^{1} \subseteq E_{\sigma^{1}}^{\kappa}$, and $S^{1}$ does not reflect at inaccessibles.

○ $S^{0} \subseteq E_{\sigma^{0}}^{\kappa}$, and $S^{0}$ does not reflect at inaccessibles.

Proof. Fix a stationary subset $T \subseteq E_{\geq \chi}^{\kappa}$ that does not reflect at inaccessibles. Since Card $(\kappa)$ is a club in the inaccessible $\kappa$, we may assume that $T \subseteq \operatorname{Card}(\kappa)$, so that $\operatorname{Tr}(T)$ is a stationary set consisting of singular cardinals. By Fodor's lemma, fix a cardinal $v \in \operatorname{Reg}(\kappa) \backslash \mu$ for which $R:=\operatorname{Tr}(T) \cap E_{v}^{\kappa}$ is stationary. As $\operatorname{Tr}(R) \subseteq \operatorname{Tr}(T)$, we can repeat the process to find $\sigma^{1} \in \operatorname{Reg}(\kappa) \backslash(v+1)$ such that $\operatorname{Tr}(R) \cap E_{\sigma^{1}}^{\kappa}$ is stationary. Now $S^{1}:=\operatorname{Tr}(R) \cap E_{\sigma^{1}}^{\kappa} \backslash\left\{\sigma^{1}\right\}$ is a stationary set consisting of singular cardinals. Repeating the process for the last time, we find $\sigma^{0} \in \operatorname{Reg}(\kappa) \backslash\left(\sigma^{1}+1\right)$ such that $S^{0}:=\operatorname{Tr}(S) \cap E_{\sigma^{0}}^{\kappa} \backslash\left\{\sigma^{0}\right\}$ is stationary. Then $\sigma^{0}>\sigma^{1}>v \geq \mu$ and $\operatorname{Tr}\left(S^{0}\right) \subseteq \operatorname{Tr}\left(S^{1}\right) \subseteq \operatorname{Tr}(T)$, so $\sigma^{1}$, $\sigma^{0}, S^{1}$ and $S^{0}$ are as sought.

Let $\sigma^{1}, \sigma^{0}, S^{1}$ and $S^{0}$ be given by the preceding claim. Note that since $S^{1}$ consists of singular cardinals, $\min \left(S^{1}\right)>\sigma_{1}$. By [Hof13, Theorem 2.1.1], we fix a sequence $\vec{e}=\left\langle e_{\delta} \mid \delta \in S^{1}\right\rangle$ such that

○ For all $\delta \in S^{1}, e_{\delta}$ is a club in $\delta$ of order type $\sigma^{1}$.

$\circ$ For all $\delta \in S^{1},\left\langle\operatorname{cf}(\gamma) \mid \gamma \in \operatorname{nacc}\left(e_{\delta}\right)\right\rangle$ is strictly increasing, converging to $\delta$.

○ For every club $D \subseteq \kappa$, there exists $\delta \in S^{1}$ with $e_{\delta} \subseteq D$.

Lemma 6.2. There exists a $C$-sequence $\vec{C}=\left\langle C_{\alpha} \mid \alpha<\kappa\right\rangle$ such that for all $\alpha<\kappa$ :

1. $\left|C_{\alpha}\right|=\operatorname{cf}(\alpha)$.

2. If $\operatorname{acc}\left(C_{\alpha}\right) \cap S^{1} \neq \emptyset$, then $\min \left(C_{\alpha}\right) \geq \operatorname{cf}(\alpha)>\sigma^{1}$.

3. For every $\delta \in\left(\operatorname{acc}\left(C_{\alpha}\right) \cup\{\alpha\}\right) \cap S^{1}, \sup \left(e_{\delta} \backslash C_{\alpha}\right)<\delta$.

Proof. This is a standard club-swallowing trick, but we do not know of a reference in which the above precise properties are exposed.

By recursion on $n<\omega$, we shall define a $C$-sequence $\vec{C}^{n}=\left\langle C_{\alpha}^{n} \mid \alpha<\kappa\right\rangle$, as follows. We commence with the case $n=0$ :

- Let $C_{0}^{0}:=\emptyset$ and $C_{\alpha+1}^{0}:=\{\alpha\}$ for all $\alpha<\kappa$.

- For each $\alpha \in \operatorname{acc}(\kappa) \backslash\left(\operatorname{Reg}(\kappa) \cup S^{1}\right)$, let $C_{\alpha}^{0}$ be a club in $\alpha$ with otp $\left(C_{\alpha}^{0}\right)=\operatorname{cf}(\alpha)=\min \left(C_{\alpha}^{0}\right)$.

- For each $\alpha \in S^{1}$, let $C_{\alpha}^{0}:=e_{\alpha} \backslash \operatorname{cf}(\alpha)$.

- For each $\alpha \in \operatorname{Reg}(\kappa)$, since $S^{1}$ consists of singular cardinals and does not reflect at inaccessibles, we may let $C_{\alpha}^{0}$ be a club in $\alpha$ with $\operatorname{acc}\left(C_{\alpha}\right) \cap S^{1}=\emptyset$.

Next, suppose that $n<\omega$ is such that $\vec{C}^{n}$ has already been defined to satisfy requirements (1) and (2) of the lemma. Define a $C$-sequence $\vec{C}^{n+1}=\left\langle C_{\alpha}^{n+1} \mid \alpha<\kappa\right\rangle$ by letting, for each $\alpha<\kappa, C_{\alpha}^{n+1}$ be the closure in $\alpha$ of the set

$$
C_{\alpha}^{n} \cup \bigcup\left\{e_{\delta} \backslash \operatorname{cf}(\alpha) \mid \delta \in \operatorname{acc}\left(C_{\alpha}^{n}\right) \cap S^{1}\right\}
$$

To see that Clauses (1) and (2) also remain valid for $\vec{C}^{n+1}$, let $\alpha<\kappa$ be arbitrary. If $C_{\alpha}^{n}=C_{\alpha}^{n+1}$, then we are done, so assume $C_{\alpha}^{n} \neq C_{\alpha}^{n+1}$. In particular, acc $\left(C_{\alpha}^{n}\right) \cap S^{1} \neq \emptyset$, so that, by the inductive hypothesis, $\left|C_{\alpha}^{n}\right|=\operatorname{cf}(\alpha)>\sigma^{1}=\operatorname{cf}(\delta)$ for all $\delta \in \operatorname{acc}\left(C_{\alpha}^{n}\right) \cap S^{1}$. In effect, $\left|C_{\alpha}^{n+1}\right|=\operatorname{cf}(\alpha)$.

Finally, for each $\alpha<\kappa$, let $C_{\alpha}$ be the closure in $\alpha$ of $\cup_{n<\omega} C_{\alpha}^{n}$. As $S^{1} \subseteq E_{\sigma^{1}}^{\kappa} \subseteq E_{>\omega}^{\kappa}$, the above construction ensures that Clause (3) holds, as well. 
Let $\vec{C}$ be given by the preceding lemma. Recalling Subsection 2.1 , we now let $\operatorname{Tr}, \operatorname{tr}, \lambda$ and $\rho_{2}$ be the characteristic functions of walking along $\vec{C}$, and let $\eta_{\alpha, \beta}$ be the notation established in Definition 2.12.

Definition 6.3. For every $(\delta, \beta) \in S^{1} \circledast \kappa$, let $\Lambda(\delta, \beta)$ denote the least $\gamma \in \operatorname{nacc}\left(e_{\delta}\right)$ such that all of the following hold:

○ $\gamma>\lambda\left(\partial_{\delta, \beta}, \beta\right)$.

$\circ \operatorname{cf}(\gamma)>\operatorname{cf}\left(\partial_{\delta, \beta}\right)$.

$\circ e_{\delta} \backslash \sup \left(e_{\delta} \cap \gamma\right) \subseteq C_{\mathrm{\partial}_{\delta, \beta}}$.

Lemma 6.4. Let $(\delta, \beta) \in S^{1} \circledast \kappa$. Then $\Lambda(\delta, \beta)$ is well-defined, and:

1. $\operatorname{nacc}\left(e_{\delta}\right) \backslash \Lambda(\delta, \beta) \subseteq \operatorname{nacc}\left(C_{\check{\delta}_{\delta, \beta}}\right)$.

2. For every $\varepsilon \in \operatorname{nacc}\left(C_{\check{\partial}_{\delta, \beta}}\right) \cap[\Lambda(\delta, \beta), \delta), \sup \left(e_{\delta} \cap \varepsilon\right) \leq \lambda(\varepsilon, \beta)<\varepsilon$.

3. For every $\varepsilon \in \operatorname{nacc}\left(C_{\partial_{\delta, \beta}}\right) \cap[\Lambda(\delta, \beta), \delta), \min \left(\operatorname{Im}(\operatorname{tr}(\varepsilon, \beta))=\mathrm{\partial}_{\delta, \beta}\right.$.

4. $\operatorname{cf}\left(\succsim_{\delta, \beta}\right) \geq \sigma^{1}$.

Proof. Since $\left\langle\operatorname{cf}(\gamma) \mid \gamma \in \operatorname{nacc}\left(e_{\delta}\right)\right\rangle$ is strictly increasing and converging to $\delta$, the first part of the following claim implies that $\Lambda(\delta, \beta)$ is well-defined.

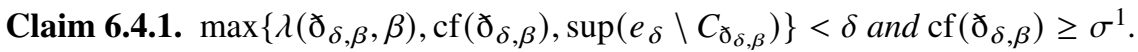

Proof. By Lemma 2.11(1), $\lambda\left(\partial_{\delta, \beta}, \beta\right)<\delta$. Now, there are two cases to consider:

- If $\delta_{\delta, \beta}=\delta$, then from $\delta \in S^{1} \subseteq E_{\sigma^{1}}^{\kappa}$ and $\min \left(S^{1}\right)>\sigma^{1}$, we infer that $\operatorname{cf}(\delta)=\sigma^{1}<\delta$. Now, by Lemma 6.2(3), $\sup \left(e_{\delta} \backslash C_{\delta}\right)<\delta$.

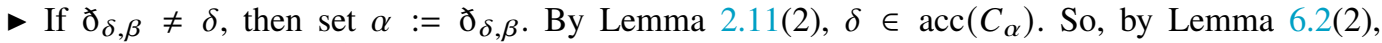
$\delta>\min \left(C_{\alpha}\right) \geq \operatorname{cf}(\alpha)>\sigma^{1}$. In addition, by Lemma 6.2(3), $\sup \left(e_{\delta} \backslash C_{\alpha}\right)<\delta$.

For every $\varepsilon \in \operatorname{nacc}\left(e_{\delta}\right)$ above $\sup \left(e_{\delta} \backslash C_{{\delta_{\beta, \delta}}}\right)$ and of cofinality greater than $\operatorname{cf}\left(\mathrm{\partial}_{\beta, \delta}\right)=\left|C_{\delta_{\beta, \delta}}\right|$, we have $\varepsilon \in \operatorname{nacc}\left(C_{\check{\beth}_{\beta, \delta}}\right)$, so that Clause (1) holds.

Now, let $\varepsilon \in \operatorname{nacc}\left(C_{\delta_{\delta, \beta}}\right) \cap[\Lambda(\delta, \beta), \delta)$ be arbitrary. We have

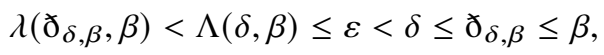

so, by Fact 2.7, $\operatorname{tr}(\varepsilon, \beta)=\operatorname{tr}\left({\partial_{\delta, \beta}}_{, \beta}\right)^{-} \operatorname{tr}\left(\varepsilon, \partial_{\delta, \beta}\right)$ and Clause (3) holds. By Lemma 2.9, $\lambda(\varepsilon, \beta)=$ $\max \left\{\lambda\left(\varlimsup_{\delta, \beta}, \beta\right), \sup \left(C_{\partial_{\delta, \beta}} \cap \varepsilon\right)\right\}$. Since $e_{\delta} \backslash \sup \left(e_{\delta} \cap \Lambda(\delta, \beta)\right) \subseteq C_{\delta_{\delta, \beta}}$, we infer that $\sup \left(C_{\delta_{\delta, \beta}} \cap \varepsilon\right) \geq$ $\sup \left(e_{\delta} \cap \varepsilon\right)$, and hence Clause (2) holds as well.

Define a collection $\mathcal{I} \subseteq \mathcal{P}(\kappa)$ via $A \in \mathcal{I}$ iff there exists a club $D \subseteq \kappa$ such that for every $\delta \in$ $S^{1} \cap \operatorname{acc}(D), \sup \left(\operatorname{nacc}\left(e_{\delta}\right) \cap D \cap A\right)<\delta$. It is clear that $\mathcal{I}$ is a $\sigma^{1}$-complete ideal over $\kappa$, extending $\mathrm{NS}_{\kappa}$. By the choice of $\vec{e}, \mathcal{I}$ is moreover proper. The next lemma is the only part of the proof that makes use of $S^{0}$ and $\sigma^{0}$.

Lemma 6.5. $\mathcal{I}$ is not weakly $\mu$-saturated, i.e., there is a partition $\kappa=\biguplus_{i<\mu} H_{i}$ such that $H_{i} \in \mathcal{I}^{+}$for every $i<\mu$.

Proof. For each $\delta \in S^{1}$, let $I_{\delta}:=\left\{A \subseteq e_{\delta} \mid \sup \left(\operatorname{nacc}\left(e_{\delta}\right) \cap A\right)<\delta\right\}$, and note:

○ $\operatorname{As} \operatorname{cf}(\delta)=\sigma^{1}, I_{\delta}$ is a $\sigma^{1}$-complete ideal over $e_{\delta}$;

- As $\sigma^{0}$ is a regular cardinal greater than $\operatorname{cf}(\delta)$, for every $\subseteq$-increasing sequence $\left\langle A_{j}\right| j\left\langle\sigma^{0}\right\rangle$ of sets from $I_{\delta}$, the union $\bigcup_{j<\sigma^{0}} A_{j}$ is in $I_{\delta}$, as well. That is, the ideal $I_{\delta}$ is $\sigma^{0}$-indecomposable.

Trivially, $\sup _{\delta \in S^{1}}\left|e_{\delta}\right|^{+}<\kappa$. Setting $\bar{C}:=\left\langle e_{\delta} \mid \delta \in S^{1}\right\rangle$ and $\bar{I}:=\left\langle I_{\delta} \mid \delta \in S^{1}\right\rangle$, and recalling [She94, Definition 3.0], it is evident that the ideal $\operatorname{id}_{p}(\bar{C}, \bar{I})$ is equal to our proper ideal $\mathcal{I}$. As $S^{0}$ is a stationary subset of $E_{\sigma^{0}}^{\kappa}$ that does not reflect at inaccessibles, Case $(\beta)(a)$ of [She94, Claim 3.3] entails the existence of a partition of $\kappa$ into $\sigma^{0}$ many $\mathcal{I}$-positive sets. In particular, since $\sigma^{0}>\mu$, $\mathcal{I}$ is not weakly $\mu$-saturated. 
By the preceding lemma, fix a surjection $h: \kappa \rightarrow \mu$ such that $H_{i}:=h^{-1}\{i\}$ is in $\mathcal{I}^{+}$for all $i<\mu$. Then, define a function $\operatorname{tr}_{h}:[\kappa]^{2} \rightarrow{ }^{<\omega} \mu$ via $\operatorname{tr}_{h}(\alpha, \beta):=h \circ \operatorname{tr}(\alpha, \beta)$.

Let $d:{ }^{<\omega} \mu \rightarrow \omega \times \mu \times \mu \times \mu$ be the function given by Fact 3.2 using $v:=\chi$. We are now ready to define our transformation.

Definition 6.6. Define $\mathbf{t}:[\kappa]^{2} \rightarrow[\kappa]^{3}$ by letting, for all $(\alpha, \beta) \in[\kappa]^{2}, \mathbf{t}(\alpha, \beta):=\left(\tau^{*}, \alpha^{*}, \beta^{*}\right)$, provided that, for $(n, i, j, \tau):=d\left(\operatorname{tr}_{h}(\alpha, \beta)\right)$, all of the following conditions are met:

○ $\beta^{*}=\operatorname{Tr}(\alpha, \beta)(n)$ is $>\alpha$,

$\circ \eta:=\lambda\left(\beta^{*}, \beta\right)$ satisfies that $\eta+1<\alpha$,

$\circ \alpha^{*}=\operatorname{Tr}(\eta+1, \alpha)\left(\eta_{\eta+1, \alpha}\right)$, and

○ $\tau^{*}=\tau<\alpha^{*}$.

Otherwise, let $\mathbf{t}(\alpha, \beta):=(0, \alpha, \beta)$.

To verify that $\mathbf{t}$ witnesses $\mathrm{P} \ell_{1}(\kappa, \mu, \chi)$, suppose that we are given a family $\mathcal{A} \subseteq[\kappa]^{<\chi}$ consisting of $\kappa$ many pairwise disjoint sets.

Lemma 6.7. For every $i<\mu$, there exist an ordinal $\zeta_{i}<\kappa$ and a sequence $\left\langle x_{\gamma} \mid \gamma \in \bar{H}_{i}\right\rangle$ such that:

- $\bar{H}_{i}$ is a stationary subset of $H_{i}$.

○ For all $\gamma \in \bar{H}_{i}, x_{\gamma} \in \mathcal{A}$ with $\min \left(x_{\gamma}\right)>\gamma$.

○ For all $\gamma \in \bar{H}_{i}$ and $\beta \in x_{\gamma}, \lambda(\gamma, \beta) \leq \zeta_{i}$.

Proof. Let $i<\mu$. By the pressing down lemma, it suffices to prove that for every club $D \subseteq \kappa$, there exist $\gamma \in D \cap H_{i}, \zeta<\gamma$ and $x \in \mathcal{A}$ with $\min (x)>\gamma$ such that $\lambda(\gamma, \beta) \leq \zeta$ for all $\beta \in x$. Thus, let $D$ be an arbitrary club in $\kappa$.

Since $H_{i}$ is in $\mathcal{I}^{+}$, we may fix $\delta \in S^{1}$ such that $\sup \left(\operatorname{nacc}\left(e_{\delta}\right) \cap D \cap H_{i}\right)=\delta$. Fix any $x \in \mathcal{A}$ with $\min (x)>\delta . \operatorname{As} \operatorname{cf}(\delta)=\sigma^{1}>|x|$, we may fix a large enough $\varepsilon \in \operatorname{nacc}\left(e_{\delta}\right) \cap D \cap H_{i}$ above $\sup _{\beta \in x} \Lambda(\delta, \beta)$. Then, by Clauses (1) and (2) of Lemma 6.4, $\sup _{\beta \in x} \lambda(\varepsilon, \beta)<\varepsilon$. So $\gamma:=\varepsilon$ and $\zeta:=\sup _{\beta \in x} \lambda(\gamma, \beta)$ are as sought.

For each $i<\mu$, let $\zeta_{i}$ and $\overrightarrow{x^{i}}=\left\langle x_{\gamma} \mid \gamma \in \bar{H}_{i}\right\rangle$ be given by the preceding lemma. Set $\zeta:=\sup _{i<\mu} \zeta_{i}$.

Definition 6.8. For $\eta<\kappa, S_{\eta}$ denotes the set of all $\epsilon<\kappa$ with the property that, for every $\varsigma<\kappa$, there exists a sequence $\left\langle\beta_{i} \mid i<\mu\right\rangle \in \prod_{i<\mu} \bar{H}_{i} \backslash \varsigma$, such that, for all $i<\mu$ and $\beta \in x_{\beta_{i}}$ :

(i) $i \in \operatorname{Im}\left(\operatorname{tr}_{h}(\epsilon, \beta)\right)$.

(ii) $\lambda(\epsilon, \beta)=\eta$.

(iii) $\rho_{2}(\epsilon, \beta)=\eta_{\epsilon, \beta}$.

Lemma 6.9. There exists $\eta<\kappa$ for which $S_{\eta}$ is stationary.

Proof. Let $D$ be an arbitrary club in $\kappa$; we shall find $\epsilon \in D$ and $\eta<\epsilon$ for which $\epsilon \in S_{\eta}$. By the choice of $\vec{e}$, the set $\Gamma:=\left\{\gamma \in S^{1} \mid \zeta<\gamma \& e_{\gamma} \subseteq D\right\}$ is stationary. Now, fix $\delta \in S^{1}$ such that $e_{\delta} \subseteq \operatorname{acc}^{+}(\Gamma)$.

Let $\varsigma<\kappa$. Fix any sequence $\left\langle\beta_{i}^{\varsigma} \mid i<\mu\right\rangle \in \prod_{i<\mu} \bar{H}_{i} \backslash \max \{\delta+1, \varsigma\}$. We shall find an ordinal $\epsilon^{\varsigma} \in D \cap \delta$, as follows.

As $\operatorname{cf}(\delta)=\sigma^{1}>\mu$, let us fix a large enough $\varepsilon^{\varsigma} \in \operatorname{nacc}\left(e_{\delta}\right)$ above $\max \left\{\zeta, \sup _{i<\mu} \Lambda\left(\delta, \beta_{i}^{\varsigma}\right)\right\}$. As $\left\langle\operatorname{cf}(\varepsilon) \mid \varepsilon \in \operatorname{nacc}\left(e_{\delta}\right)\right\rangle$ is strictly increasing and converging to $\delta$, we may also require that $\operatorname{cf}\left(\varepsilon^{\varsigma}\right)>\mu$. By Lemma 6.4(2), $\Lambda^{\varsigma}:=\max \left\{\zeta, \sup _{i<\mu} \lambda\left(\varepsilon^{\varsigma}, \beta_{i}^{\varsigma}\right)\right\}$ is smaller than $\varepsilon^{\varsigma}$. As $\varepsilon^{\varsigma} \in \operatorname{nacc}\left(e_{\delta}\right) \subseteq \operatorname{acc}^{+}(\Gamma)$, let us pick $\gamma^{\varsigma} \in \Gamma$ with $\Lambda^{\varsigma}<\gamma^{\varsigma}<\varepsilon^{\varsigma}$. Now, fix a large enough $\epsilon^{\varsigma} \in \operatorname{nacc}\left(e_{\gamma^{\varsigma}}\right) \subseteq D \cap \delta$ to satisfy $\sup \left(e_{\gamma^{\varsigma}} \cap \epsilon^{\varsigma}\right)>\max \left\{\Lambda^{\varsigma}, \Lambda\left(\gamma^{\varsigma}, \varepsilon^{\varsigma}\right)\right\}$. Denote $\alpha^{\varsigma}:=\mathrm{\partial}_{\gamma^{\varsigma}, \varepsilon^{\varsigma}}$.

By the pigeonhole principle, let us fix $\epsilon \in D \cap \delta$, and $\eta \leq \epsilon$ for which

$$
\Sigma:=\left\{\varsigma<\kappa \mid \epsilon^{\varsigma}=\epsilon \& \sup \left(C_{\alpha} \varsigma \cap \epsilon^{\varsigma}\right)=\eta\right\}
$$

is cofinal in $\kappa$. We already know that $\epsilon \in D$; we shall later show that $\eta<\epsilon$. 
To see that $\epsilon \in S_{\eta}$, let $\varsigma<\kappa$ be arbitrary. By increasing $\varsigma$, we may assume that $\varsigma \in \Sigma$. Let $i<\mu$ and $\beta \in x_{\beta_{i}^{\varsigma}}$ be arbitrary. We shall show that:

(i') $\operatorname{tr}(\epsilon, \beta)=\operatorname{tr}\left(\beta_{i}^{S}, \beta\right)^{-} \operatorname{tr}\left(\epsilon, \beta_{i}^{S}\right)$.

(ii') $\lambda(\epsilon, \beta)=\eta$.

(iii') $\rho_{2}(\epsilon, \beta)=\eta_{\epsilon, \beta}$.

We have:

$$
\max \left\{\lambda\left(\beta_{i}^{\varsigma}, \beta\right), \lambda\left(\varepsilon^{\varsigma}, \beta_{i}^{\varsigma}\right)\right\} \leq \max \left\{\Lambda^{\varsigma}, \Lambda\left(\gamma^{\varsigma}, \varepsilon^{\varsigma}\right)\right\}<\epsilon<\gamma^{\varsigma}<\varepsilon^{\varsigma}<\delta<\beta_{i}^{\varsigma}<\beta .
$$

It thus follows from Fact 2.7 that Clause (i') is satisfied, so that $i \in \operatorname{Im}\left(\operatorname{tr}_{h}(\epsilon, \beta)\right)$. It also follows from Fact 2.7 that $\operatorname{tr}\left(\epsilon, \beta_{i}^{S}\right)=\operatorname{tr}\left(\varepsilon^{S}, \beta_{i}^{S}\right)^{-} \operatorname{tr}\left(\epsilon, \varepsilon^{S}\right)$. In addition, by Clauses (1) and (3) of Lemma 6.4, $\operatorname{tr}\left(\epsilon, \varepsilon^{\varsigma}\right)=\operatorname{tr}\left(\alpha^{\varsigma}, \varepsilon^{\varsigma}\right)^{-} \operatorname{tr}\left(\epsilon, \alpha^{\varsigma}\right)$. Thus, altogether:

$$
\operatorname{tr}(\epsilon, \beta)=\operatorname{tr}\left(\beta_{i}^{\varsigma}, \beta\right)^{-} \operatorname{tr}\left(\varepsilon^{\varsigma}, \beta_{i}^{\varsigma}\right)^{-} \operatorname{tr}\left(\alpha^{\varsigma}, \varepsilon^{\varsigma}\right)^{-} \operatorname{tr}\left(\epsilon, \alpha^{\varsigma}\right) .
$$

As $\epsilon$ is an element of nacc $\left(e_{\gamma \varsigma}\right)$ above $\Lambda\left(\gamma^{\varsigma}, \varepsilon^{\varsigma}\right) \geq \sup \left(e_{\gamma \varsigma} \backslash C_{\alpha \varsigma}\right)$, we infer from Lemma 6.4(1) that $\epsilon \in \operatorname{nacc}\left(C_{\alpha^{\varsigma}}\right)$ and hence $\lambda\left(\epsilon, \alpha^{\varsigma}\right)=\sup \left(C_{\alpha^{\varsigma}} \cap \epsilon\right)$. As $\epsilon=\epsilon^{\varsigma}$, it follows from Lemma 6.4(2) that

$$
\begin{aligned}
\max \left\{\lambda\left(\beta_{i}^{\varsigma}, \beta\right), \lambda\left(\varepsilon^{\varsigma}, \beta_{i}^{\varsigma}\right), \lambda\left(\alpha^{\varsigma}, \varepsilon^{\varsigma}\right)\right\} & \leq \max \left\{\Lambda^{\varsigma}, \Lambda\left(\gamma^{\varsigma}, \varepsilon^{\varsigma}\right)\right\} \\
& <\sup \left(e_{\gamma} \cap \cap\right) \\
& \leq \sup \left(C_{\alpha} \cap \epsilon\right) \\
& =\eta
\end{aligned}
$$

Altogether, $\lambda(\epsilon, \beta)=\sup \left(C_{\alpha \varsigma} \cap \epsilon\right)=\eta$ and $\rho_{2}(\epsilon, \beta)=\eta_{\epsilon, \beta}$. In addition, since $\eta=\sup \left(C_{\alpha \varsigma} \cap \epsilon\right)$ and $\epsilon \in \operatorname{nacc}\left(C_{\alpha} \varsigma\right)$, we infer that $\eta<\epsilon$, as promised.

Let $\eta$ be given by the preceding lemma. Let $D$ be a club in $\kappa$ such that for all $\delta \in D$, there exists $M_{\delta} \prec \mathcal{H}_{\kappa^{+}}$containing the parameter $p:=\left\{S_{\eta},\left\langle\overrightarrow{x^{i}} \mid i<\mu\right\rangle, \vec{C}, h\right\}$ and satisfying $M_{\delta} \cap \kappa=\delta$. For every $j<\mu$, since $H_{j}$ is in $\mathcal{I}^{+}$, the set $\Delta_{j}:=\left\{\delta \in S^{1} \mid \sup \left(\operatorname{nacc}\left(e_{\delta}\right) \cap D \cap H_{j}\right)=\delta\right\}$ is stationary. Finally, let

$$
S^{*}:=S_{\eta} \cap \bigcap_{j<\mu} \operatorname{acc}^{+}\left(\Delta_{j}\right) .
$$

Lemma 6.10. Let $\left(\tau^{*}, \alpha^{*}, \beta^{*}\right) \in \mu \circledast S^{*} \circledast S^{*}$. There exists $(a, b) \in[\mathcal{A}]^{2}$ such that $\mathbf{t}[a \times b]=$ $\left\{\left(\tau^{*}, \alpha^{*}, \beta^{*}\right)\right\}$.

Proof. While reading the upcoming proof, the reader may want to consult with Figure 2 below. As $\beta^{*} \in S^{*} \subseteq S_{\eta}$, let us fix a sequence $\left\langle\beta_{i} \mid i<\mu\right\rangle \in \prod_{i<\mu} \bar{H}_{i} \backslash\left(\beta^{*}+1\right)$ such that for all $i<\mu$ and $\beta \in x_{\beta_{i}}$ :

1. $i \in \operatorname{Im}\left(\operatorname{tr}_{h}\left(\beta^{*}, \beta\right)\right)$.

2. $\lambda\left(\beta^{*}, \beta\right)=\eta$.

3. $\rho_{2}\left(\beta^{*}, \beta\right)=\eta_{\beta^{*}, \beta}$.

For all $j<\mu$, as $\beta^{*} \in \operatorname{acc}^{+}\left(\Delta_{j}\right)$, we may pick $\delta_{j} \in \Delta_{j} \cap \beta^{*}$ above $\alpha^{*}$, so that $\delta_{j}>\alpha^{*}>\eta$. Now, pick $\varepsilon_{j} \in \operatorname{nacc}\left(e_{\delta_{j}}\right) \cap D \cap H_{j}$ above $\max \left\{\alpha^{*}, \Lambda\left(\delta_{j}, \beta^{*}\right)\right\}$. As $M_{\varepsilon_{j}}$ contains $p$, we have that $S_{\eta} \in M_{\varepsilon_{j}}$. Now, by Clauses (1) and (2) of Lemma 6.4, $\varsigma_{j}:=\max \left\{\alpha^{*}, \Lambda\left(\delta_{j}, \beta^{*}\right), \lambda\left(\varepsilon_{j}, \beta^{*}\right)\right\}+1$ is smaller than $\varepsilon_{j}$. Since $\alpha^{*} \in M_{\varepsilon_{j}} \cap S_{\eta}$, we may then find $\alpha_{j} \in M_{\varepsilon_{j}} \cap \bar{H}_{j} \backslash \varsigma_{j}$ such that for all $\alpha \in x_{\alpha_{j}}$ :

(2') $\lambda\left(\alpha^{*}, \alpha\right)=\eta$.

(3') $\rho_{2}\left(\alpha^{*}, \alpha\right)=\eta_{\alpha^{*}, \alpha}$.

Note that from $\alpha_{j} \in M_{\varepsilon_{j}}$, it follows that $\sup \left(x_{\alpha_{j}}\right)<\varepsilon_{j}$. Write $a_{j}:=x_{\alpha_{j}}$ and $b_{i}:=x_{\beta_{i}}$. Fix arbitrary $(i, j) \in[\mu]^{2}$ and $(\alpha, \beta) \in a_{j} \times b_{i}$. Then:

$$
\eta+1<\alpha^{*} \leq \max \left\{\alpha^{*}, \lambda\left(\beta^{*}, \beta\right), \lambda\left(\varepsilon_{j}, \beta^{*}\right)\right\}<\varsigma_{j} \leq \alpha_{j}<\alpha<\varepsilon_{j}<\beta^{*}<\beta_{i}<\beta .
$$




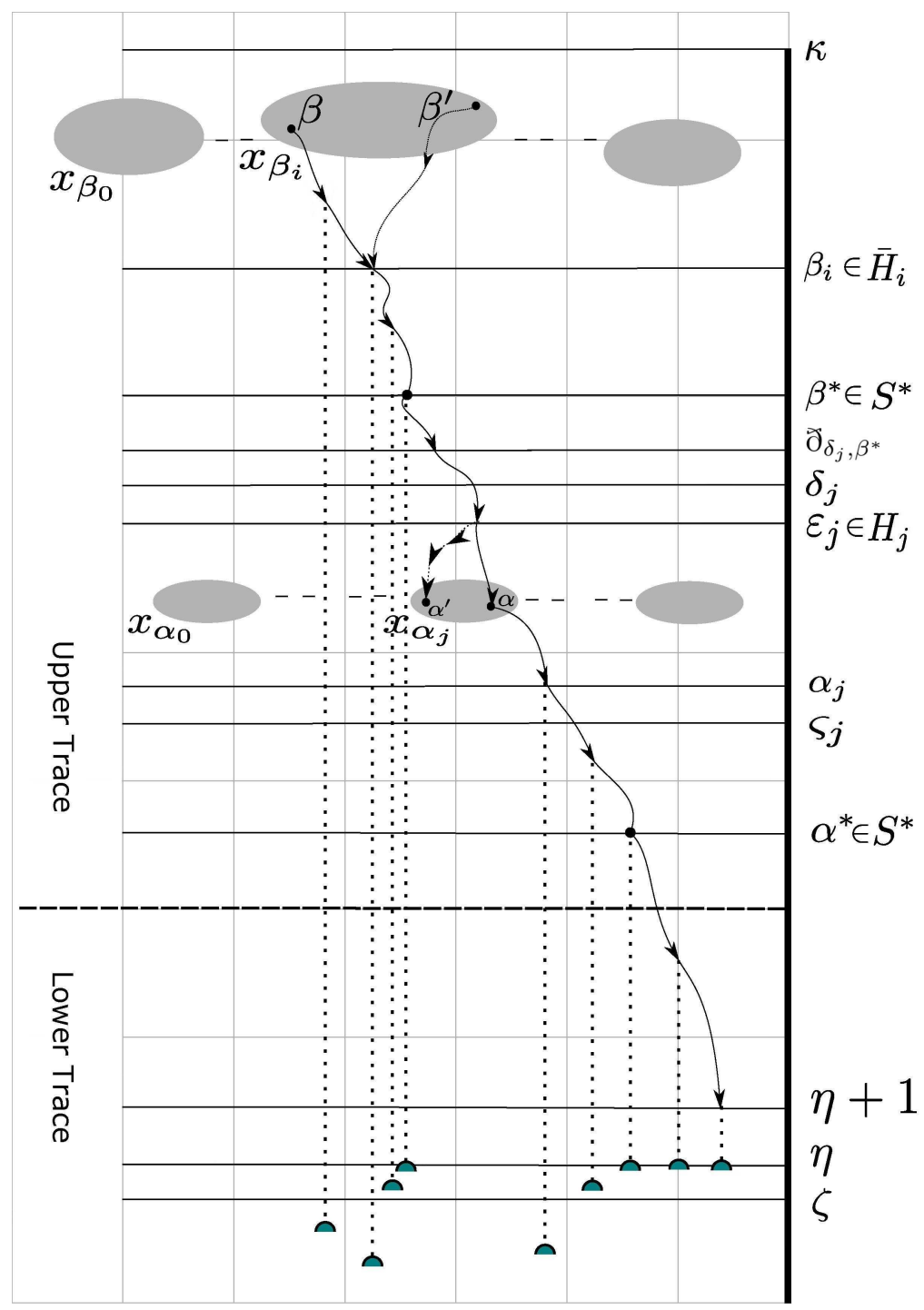

Figure 2. Illustration of the proof of Lemma 6.10.

So, by Fact 2.7:

$$
\operatorname{tr}(\alpha, \beta)=\operatorname{tr}\left(\beta^{*}, \beta\right)^{-} \operatorname{tr}\left(\varepsilon_{j}, \beta^{*}\right)^{-} \operatorname{tr}\left(\alpha, \varepsilon_{j}\right) .
$$

For each $i<\mu$, set $u_{i}:=\left\{\operatorname{tr}_{h}\left(\beta^{*}, \beta\right) \mid \beta \in b_{i}\right\}$. By Clause (1) above, $i \in \operatorname{Im}(\varrho)$ for all $\varrho \in u_{i}$. For each $j\left\langle\mu\right.$, set $v_{j}:=\left\{\operatorname{tr}_{h}\left(\alpha, \beta^{*}\right) \mid \alpha \in a_{j}\right\}$ and $\sigma_{j}:=\operatorname{tr}_{h}\left(\varepsilon_{j}, \beta^{*}\right)$. As $\varepsilon_{j} \in H_{j}$, we infer that $\sigma_{j}-\langle j\rangle \sqsubseteq \sigma$ for all $\sigma \in v_{j}$.

Finally, by the choice of $d$, fix $(i, j) \in[\mu]^{2}$ such that $d\left(\varrho^{-} \sigma\right)=\left(\ell(\varrho), i, j, \tau^{*}\right)$ for all $\varrho \in u_{i}$ and $\sigma \in v_{j}$. Set $a:=a_{j}$ and $b:=b_{i}$, so that $(a, b) \in[\mathcal{A}]^{2}$.

To see that $\mathbf{t}[a \times b]=\left\{\left(\tau^{*}, \alpha^{*}, \beta^{*}\right)\right\}$, fix arbitrary $\alpha \in a$ and $\beta \in b$. Denote $\varrho:=\operatorname{tr}_{h}\left(\beta^{*}, \beta\right)$ and $\sigma:=\operatorname{tr}_{h}\left(\alpha, \beta^{*}\right)$, so that $\varrho \in u_{i}$ and $\sigma \in v_{j}$. Denote $\left(n, i^{\prime}, j^{\prime}, \tau\right):=d\left(\operatorname{tr}_{h}(\alpha, \beta)\right)$. Then:

○ $\operatorname{Tr}(\alpha, \beta)(n)=\operatorname{Tr}(\alpha, \beta)\left(\rho_{2}\left(\beta^{*}, \beta\right)\right)=\beta^{*}$.

$\circ \tau=\tau^{*}$.

$\circ=\lambda\left(\beta^{*}, \beta\right)$ and $\eta+1<\alpha$.

$\circ \tau^{*}<\mu<\alpha^{*}$. 
Now, since $\lambda\left(\alpha^{*}, \alpha\right)=\eta<\eta+1<\alpha^{*}<\alpha, \operatorname{tr}(\eta+1, \alpha)=\operatorname{tr}\left(\alpha^{*}, \alpha\right)^{-} \operatorname{tr}\left(\eta+1, \alpha^{*}\right)$. So, since $\rho_{2}\left(\alpha^{*}, \alpha\right)=\eta_{\alpha^{*}, \alpha}, \rho_{2}\left(\alpha^{*}, \alpha\right)=\eta_{\eta+1, \alpha}$ and $\alpha^{*}=\operatorname{Tr}(\eta+1, \alpha)\left(\eta_{\eta+1, \alpha}\right)$.

Acknowledgement. The results of this paper were presented by the first author in a talk at the Oberseminar Mengenlehre at Universität Münster, January 2020. He thanks the hosts for the warm hospitality and the participants of the seminar for their feedback.

The authors are grateful to the anonymous referee for a thorough reading of the paper and for providing a thoughtful list of corrections.

\section{Conflict of Interest: None.}

Financial support. The first author is partially supported by the European Research Council (grant agreement ERC-2018StG 802756) and by the Israel Science Foundation (grant agreement 2066/18). The second author is supported by the Foreign Postdoctoral Fellowship Program of the Israel Academy of Sciences and Humanities and by the Israel Science Foundation (grant agreement 2066/18).

\section{References}

[BR19] Ari Meir Brodsky and Assaf Rinot. Distributive Aronszajn trees. Fund. Math., 245(3): 217-291, 2019.

[Eis13a] Todd Eisworth. Getting more colors I. J. Symbolic Logic, 78(1): 1-16, 2013.

[Eis13b] Todd Eisworth. Getting more colors II. J. Symbolic Logic, 78(1): 17-38, 2013.

[FR17] David Fernandez-Breton and Assaf Rinot. Strong failures of higher analogs of Hindman's theorem. Trans. Amer. Math. Soc., 369(12): 8939-8966, 2017.

[Hin74] Neil Hindman. Finite sums from sequences within cells of a partition of N. J. Combinatorial Theory Ser. A, 17: $1-11,1974$.

[HLS17] Neil Hindman, Imre Leader, and Dona Strauss. Pairwise sums in colourings of the reals. Abh. Math. Semin. Univ. Hambg., 87(2): 275-287, 2017.

[Hof13] Douglas J. Hoffman. A Coloring Theorem for Inaccessible Cardinals. ProQuest LLC, Ann Arbor, MI, 2013.

[IR21] Tanmay Inamdar and Assaf Rinot. Was Ulam right? http://assafrinot.com/paper/47, 2021. In preparation.

[LHR21] Chris Lambie-Hanson and Assaf Rinot. Knaster and friends II: The C-sequence number. J. Math. Log., 21(1):2150002, 54, 2021.

[Ram30] F.P. Ramsey. On a problem of formal logic. Proc. London Math. Soc., pages 264-286, 1930.

[Rin12] Assaf Rinot. Transforming rectangles into squares, with applications to strong colorings. Adv. Math., 231(2): 1085-1099, 2012.

[Rin14a] Assaf Rinot. Chain conditions of products, and weakly compact cardinals. Bull. Symb. Log., 20(3): 293-314, 2014.

[Rin14b] Assaf Rinot. Complicated colorings. Math. Res. Lett., 21(6): 1367-1388, 2014.

[Rin17] Assaf Rinot. Higher Souslin trees and the GCH, revisited. Adv. Math., 311(C): 510-531, 2017.

[RT13] Assaf Rinot and Stevo Todorcevic. Rectangular square-bracket operation for successor of regular cardinals. Fund. Math., 220( 2): 119-128, 2013.

[RZ21] Assaf Rinot and Jing Zhang. Strongest transformations. http://assafrinot.com/paper/45, 2021. In preparation.

[She88] Saharon Shelah. Successors of singulars, cofinalities of reduced products of cardinals and productivity of chain conditions. Israel J. Math., 62(2): 213-256, 1988.

[She94] Saharon Shelah. There are Jonsson algebras in many inaccessible cardinals. In Cardinal Arithmetic, volume 29 of Oxford Logic Guides. Oxford University Press, 1994.

[She97] Saharon Shelah. Colouring and non-productivity of $\boldsymbol{\aleph}_{2}$-cc. Annals of Pure and Applied Logic, 84: 153-174, 1997.

[Sie33] Waclaw Sierpiński. Sur un problème de la théorie des relations. Ann. Scuola Norm. Sup. Pisa Cl. Sci. (2), 2(3): 285-287, 1933.

[SS10] Daniel Soukup and Lajos Soukup. Club guessing for dummies. arXiv preprint arXiv:1003.4670, 2010.

[Tod87] Stevo Todorčević. Partitioning pairs of countable ordinals. Acta Math., 159(3-4): 261-294, 1987.

[Tod07] Stevo Todorcevic. Walks on ordinals and their characteristics, volume 263 of Progress in Mathematics. Birkhäuser Verlag, Basel, 2007. 